NBER WORKING PAPER SERIES

\title{
ESTIMATING DYNAMIC DISCRETE CHOICE MODELS WITH HYPERBOLIC DISCOUNTING, WITH AN APPLICATION TO MAMMOGRAPHY DECISIONS
}

\author{
Hanming Fang \\ Yang Wang \\ Working Paper 16438 \\ http://www.nber.org/papers/w16438
NATIONAL BUREAU OF ECONOMIC RESEARCH
1050 Massachusetts Avenue
Cambridge, MA 02138
October 2010

We would like to thank Peter Arcidiacono, Patrick Bayer, Han Hong, Joe Hotz, Edward Kung, Thierry Magnac, Aprajit Mahajan, Ted O'Donoghue, Dan Silverman, Frank Sloan, Xun Tang and seminar/conference participants at Cornell, AEA Meetings in San Francisco (2009) and Midwest Health Economics Conference in Chicago (2010) for helpful comments and suggestions. The first draft of the paper (January 2009) was completed when both authors were at Duke University. Fang gratefully acknowledges financial support from NSF Grant SES 0844845. We are responsible for all remaining errors. The views expressed herein are those of the authors and do not necessarily reflect the views of the National Bureau of Economic Research.

NBER working papers are circulated for discussion and comment purposes. They have not been peerreviewed or been subject to the review by the NBER Board of Directors that accompanies official NBER publications.

(C) 2010 by Hanming Fang and Yang Wang. All rights reserved. Short sections of text, not to exceed two paragraphs, may be quoted without explicit permission provided that full credit, including $\odot$ notice, is given to the source. 
Estimating Dynamic Discrete Choice Models with Hyperbolic Discounting, with an Application to Mammography Decisions

Hanming Fang and Yang Wang

NBER Working Paper No. 16438

October 2010

JEL No. C14,I1

\begin{abstract}
We extend the semi-parametric estimation method for dynamic discrete choice models using Hotz and Miller's (1993) conditional choice probability (CCP) approach to the setting where individuals may have hyperbolic discounting time preferences and may be naive about their time inconsistency. We illustrate the proposed estimation method with an empirical application of adult women's decisions to undertake mammography to evaluate the importance of present bias and naivety in the under-utilization of this preventive health care. Our results show evidence for both present bias and naivety.
\end{abstract}

\author{
Hanming Fang \\ Department of Economics \\ University of Pennsylvania \\ 3718 Locust Walk \\ Philadelphia, PA 19104 \\ and NBER \\ hanming.fang@econ.upenn.edu \\ Yang Wang \\ Department of Economics \\ Lafayette College \\ 213 Simon Center \\ Easton, PA 18042 \\ wangy@lafayette.edu
}




\section{Introduction}

Dynamic discrete choice models have been used to understand a wide range of economic behavior. The early dynamic discrete choice models that are empirically implemented tend to be parametric; ${ }^{1}$ but recently, a growing list of authors have addressed the non- or semi-parametric identification of dynamic discrete choice models. The earliest attempt in this regard is Hotz and Miller (1993) which pioneered the approach of using conditional choice probabilities to infer about choice-specific continuation values. Rust (1994a, 1994b) showed that the discount factor in standard dynamic discrete choice models are generically not identified; Magnac and Thesmar (2002) expanded Rust's non-identification results, and proposed exclusion restrictions that lead to the identification of the standard discount factor.

All of the above-mentioned literature model the impatience of the decision makers by assuming that agents discount future streams of utility or profits exponentially over time. As is now well known, exponential discounting is not just an analytically convenient assumption; without this assumption, intertemporal marginal rates of substitution will change as time passes, and preferences will be time-inconsistent [see Strotz (1956, p.172)]. A recent theoretical literature has built on the work of Strotz (1956) and others to explore the consequences of relaxing the standard assumption of exponential discounting. Drawing both on experimental research and on common intuition, economists have built models of quasi-hyperbolic discounting to capture the tendency of decision makers to seize short-term rewards at the expense of long-term preferences. ${ }^{2}$ This literature studies the implications of time-inconsistent preferences, and their associated problems of self-control, for a variety of economic choices and environments. ${ }^{3}$

A small list of empirical papers that attempted to estimate dynamic models with hyperbolic discounting time preferences have followed the parametric approach (Fang and Silverman 2009, Laibson, Repetto and Tobacman 2007 and Paserman 2008). ${ }^{4}$ Fang and Silverman (2009) empiri-

\footnotetext{
${ }^{1}$ The earliest formulation and estimation of parametric dynamic discrete choice models include Wolpin (1984) for fertility choice, Miller (1984) for occupational choice, Pakes (1986) for patent renewal, and Rust (1987) for bus engine replacement.

${ }^{2}$ A body of experimental research, reviewed in Ainslie (1992) and Loewenstein and Elster (1992), indicates that hyperbolic time discounting may parsimoniously explain some basic features of the intertemporal decision making that are inconsistent with simple models with exponential discounting. Specifically, standard decision models with exponential discounting are not easy to reconcile with commonly observed preference reversals: subjects choose the larger and later of two prizes when both are distant in time, but prefer the smaller but earlier one as both prizes draw nearer to the present (see Rubinstein 2003, however, for an alternative explanation of preference reversals).

${ }^{3}$ For example, models of time-inconsistent preferences have been applied by Laibson (1997) and O'Donoghue and Rabin (1999a,b) to consumption and savings; by Barro (1999) to growth; by Gruber and Koszegi (2001) to smoking decisions; by Krusell, Kuruşçu, and Smith (2002) to optimal tax policy; by Carrillo and Mariotti (2000) to belief formation; by Fang and Silverman (2004) to welfare program participation and labor supply of single mothers with dependent children; and by Della Vigna and Paserman (2005) to job search.

${ }^{4}$ Also related, Arcidiacono, Sieg and Sloan (2007) estimate a parametric forward-looking dynamic discrete choice model of smoking and heavy drinking for late-middle age men in the Health and Retirement Studey (HRS) and find
} 
cally implement a dynamic structural model of labor supply and welfare program participation for never-married mothers with potentially time-inconsistent preferences. Using panel data on the choices of single women with children from the National Longitudinal Survey of Youth (NLSY 1979), they provide estimates of the degree of time-inconsistency, and of its influence on the welfare take-up decision. For the particular population of single mothers with dependent children, they estimate the present bias factor and the standard discount factor to be 0.338 and 0.88 respectively, implying a one-year ahead discount rate of 238\%. Laibson, Repetto and Tobacman (2007) use the Method of Simulated Moments (MSM) to estimate time preferences - both short and long run discount rates - from a structural buffer stock consumption model that includes many realistic features such as stochastic labor income, liquidity constraints, child and adult dependents, liquid and illiquid assets, revolving credit and retirement. Under parametric assumptions on the model, the model is identified from matching the model's predictions of retirement wealth accumulation, credit card borrowing and consumption-income co-movement with those observed in the data. Their benchmark estimates imply a $48.5 \%$ short-term annualized discount rate and a $4.3 \%$ long-term annualized discount rate. Paserman (2008) estimates the structural parameters of a job search model with hyperbolic discounting and endogenous search effort, using data on duration of unemployment spells and accepted wages from the NLSY 1979. Under parametric assumptions of the model, identification of the hyperbolic discounting parameters comes from the variation in the relative magnitude of unemployment duration and accepted wages. Indeed he finds that the results are sensitive to the specific structure of the model and on the functional form assumption for the distribution of offered wages. For low-wage workers, he rejects the exponential discounting model and estimates a one-year discount rate of about $149 \% .{ }^{5}$ Chung, Steenburgh and Sudhir (2009) estimated a dynamic structural model of sales force response to a bonus-based compensation plan where the salesman might have hyperbolic discounting time preferences. Exploiting the bonus-based compensation structure, they found some evidence consistent with present bias.

None of the above papers allow for the possibility that a hyperbolic discounting decisionmaker may also be naive. More importantly, the identification of the present bias and standard discount factors in these papers are often based on parametric assumptions imposed on the model. To the best of our knowledge, it is not known whether dynamic discrete choice models with hyperbolic discounting preferences can be semi-parametrically identified using standard short-panel

that a forward-looking model fits the data (mainly the age profile for heavy-drinking and smoking) better than a myopic model. Their model assumes exponential discounting and thus does not incorporate the possibility that time inconsistent preferences may play a role in the consumption of alcohol and cigarettes.

${ }^{5}$ There are other inferential studies about discount rates that exploit specific clear-cut intertemporal trade-offs. For example, Hausman (1979), and Warner and Pleeter (2001) estimate discount rates ranging from 0 to $89 \%$ depending on the characteristics of the individual and intertemporal trade-offs at stake. 
data that are typically used in these papers. ${ }^{6,7}$

In this paper, we consider a standard dynamic discrete choice model where decision makers potentially exhibit hyperbolic discounting preferences in the form of a present-bias factor $(\beta)$, an exponential discounting factor $(\delta)$ and a potential naivety parameter $(\tilde{\beta})$ [as in $\mathrm{O}^{\prime}$ Donoghue and Rabin (1999a)], and examine the conditions under which the primitive parameters of the model, including the three hyperbolic discounting time preference parameters, can be identified using short-panel (two periods) data. We show that, if there exist exclusion variables that affect the transition probabilities of states over time but do not affect the decision-makers' static payoff functions, a condition similar to that in Magnac and Thesmar (2002) necessary for the identification of dynamic discrete choice models with standard exponential discounting, then we can potentially identify all three discount factors $\beta, \tilde{\beta}$ and $\delta$.

The intuition for why exclusion variables that affect the transition of state variables but not static payoffs might provide source of identification for the discount factors can be described as follows. Consider two decision-makers who share the same period-payoff relevant state variables but differ only in the exclusion variables. Because the exclusion variables only affect the transition of the payoff-relevant state variables, their effects on the choices in the current period will inform us about the degree to which the agents discount the future. The intuition for why $\beta, \tilde{\beta}$ and $\delta$ can be separately identified will be provided later in Section 3.2.

We propose two estimation approaches that are intimately related to our identification arguments. One approach is based on maximizing a pseudo-likelihood function and the other is based on minimizing the estimated variation of the static payoff functions with respect to the exclusion variables. Monte Carlo experiments show that both estimators perform well in large samples, but in relatively small samples, the maximum pseudo-likelihood based estimator performs better. We thus use maximum pseudo-likelihood based estimator in our empirical application.

Our paper also represents an interesting intermediate case between the literature on estimating dynamic discrete choice single-agent decision problems [see Miller (1984), Wolpin (1984), Pakes (1986), Rust (1987), Hotz and Miller (1993) for early contributions and Rust (1994a, 1994b) and Aguirregabiria and Mira (2007b) for surveys] and the more recent literature on estimating dynamic games [see Pakes and McGuire (1994), Pesendorfer and Schmidt-Dengler (2003), Pakes, Ostrovsky and Berry (2007), Aguirregabiria and Mira (2007a), Bajari, Benkard and Levin (2007), among others; and see Bajari, Hong and Nekipelov (2010) for a survey]. As is well-known, if an agent has hyperbolic discounting time preferences, the outcome of her decision process can be

\footnotetext{
${ }^{6}$ Fang and Silverman (2006) is an exception. They argue that exponential discounting and hyperbolic discounting models are distinguishable, using an argument based on observed choice probabilities.

${ }^{7}$ Mahajan and Tarozzi (2010) discuss how data about expectations regarding future behavior might be used to identify and estimate hyperbolic discounting parameters in their study of the adoption of insecticide treated nets.
} 
considered as the equilibrium outcome of an intra-personal game with the players being the selves of the same individual at different periods. There are two crucial differences, however, between the intra-personal games we analyze for agents with time-inconsistent time preferences and those in the existing dynamic games literature. The first difference is that in the intra-personal game case, we do not observe the actions of all the players. More specifically, the outcomes - choices and the evolutions of the state variables - we observe in the data are affected only by the current selves, even though the current selves' choices are impacted by their perception of future selves' actions. Secondly, the dynamic games literature [e.g. Bajari, Benkard and Levin 2007] may allow for the different players to have different period-payoff functions, however, in our setting the payoffs for the players - the current self and the future selves - differ only in time preferences; moreover, under hyperbolic discounting, we are assuming a rather restricted form of time preference differences between the players.

We illustrate our identification argument and estimation method with an empirical application of adult women's decisions to undertake mammography to investigate the role of time inconsistent preferences in the under-utilization of this preventive care. We consider a simple model where mammography can potentially lower the probability of death in the next two years and it may also lower the probability of bad health conditional on surviving in two years; however, undertaking mammography may involve immediate costs (most of which we would like to interpret as psychological and physical costs instead of financial costs). For the purpose of identifying the hyperbolic discounting preference parameters, we use several variables, including the indicator for either the woman's mother is still alive and/or whether she died at age greater than 70, as the exclusion variables that do not enter the relevant instantaneous payoff function but affects the transition probability of other instantaneous payoff-relevant state variables. Our estimates indicate that individuals exhibit both present bias and naivety as $\beta$ and $\tilde{\beta}$ are estimated to be about 0.72 and 0.99 , respectively, suggesting both present bias $(\beta<1)$ and naivety $(\tilde{\beta}>\beta)$. These suggest that both present bias and naivety might have played an important role in the fact that nearly $25 \%$ of the women do not undertake mammography as advised by American Cancer Association, which is universally regarded as a very cost effective way for early detection of breast cancer (see Degnan et al. 1992).

The remainder of the paper is structured as follows. In Section 2 we describe a general dynamic discrete choice model with hyperbolic discounting time preferences. In Section 3 we provide detailed analysis for identification. In Section 4, we propose two estimation strategies based on the identification arguments for the discount factors and we also evaluate the performance of our proposed estimation methods using Monte Carlo experiments. In Section 5 we provide the background information for mammography, which is the decision we examine in our empirical 
application; we also describe the data set used in our study and provide some basic descriptive statistics of the samples; we then provide details about the empirical specification of our model of the decision for undertaking mammography and present the main estimation results. Finally, Section 6 concludes and discusses a few important issues abstracted away in our analysis. In the Appendix, we provide additional details about how our identification argument can be extended to finite horizon applications.

\section{Dynamic Discrete Choice Model with Hyperbolic Discounting Time Preferences}

\subsection{Basic Model Setup}

Consider a decision maker whose intertemporal utility is additively time separable. The agent's instantaneous preferences are defined over the action she chooses from a discrete set of alternatives $i \in \mathcal{I}=\{0,1, \ldots, I\}$, and a list of state variables denoted by $h \equiv(x, \varepsilon)$ where $x \in \mathcal{X}$, which for notational simplicity includes time $t$, are observed by the researcher, and $\varepsilon \equiv\left(\varepsilon_{1}, \ldots, \varepsilon_{I}\right) \in R^{I}$ are the vector of random preference shocks for each of the $I$ alternatives. ${ }^{8}$ We make the following assumption about the instant utility from taking action $i, u_{i}^{*}(h) \equiv u_{i}^{*}(x, \varepsilon)$ :

Assumption 1. (Additive Separability) The instantaneous utilities are given by, for each $i \in \mathcal{I}$,

$$
u_{i}^{*}(x, \varepsilon)=u_{i}(x)+\varepsilon_{i},
$$

where $u_{i}(x)$ is the deterministic component of the utility from choosing $i$ at $x$, and $\left(\varepsilon_{1}, \ldots, \varepsilon_{I}\right)$ has a joint distribution $G$, which is absolutely continuous with respect to the Lebesgue measure in $R^{I}$.

We assume that the time horizon is infinite with time denoted by $t=1,2, \ldots$ The decisionmaker's intertemporal preferences are represented by a simple and now commonly used formulation of agents' potentially time-inconsistent preferences: $(\beta, \delta)$-preferences (Phelps and Pollak, 1968; Laibson, 1997; and O’Donoghue and Rabin 1999a):

Definition 1. $(\beta, \delta)$-preferences are intertemporal preferences represented by

$$
U_{t}\left(u_{t}, u_{t+1}, \ldots\right) \equiv u_{t}+\beta \sum_{k=t+1}^{+\infty} \delta^{k-t} u_{k}
$$

where $\beta \in(0,1]$ and $\delta \in(0,1]$.

\footnotetext{
${ }^{8}$ We assume that $\mathcal{X}$ is a finite set and denote $X=\# \mathcal{X}$ to be the size of the state space.
} 
Following the terminology of O'Donoghue and Rabin (1999a), the parameter $\delta$ is called the standard discount factor, which captures long-run, time-consistent discounting; and the parameter $\beta$ is called the present-bias factor, which captures short-term impatience. The standard model is nested as a special case of $(\beta, \delta)$-preferences when $\beta=1$. When $\beta \in(0,1),(\beta, \delta)$-preferences capture "quasi-hyperbolic" time discounting (Laibson, 1997). We say that an agent's preferences are time-consistent if $\beta=1$, and are present-biased if $\beta \in(0,1)$.

The literature on time-inconsistent preferences distinguishes between naive and sophisticated agents (Strotz, 1956; Pollak, 1968; O'Donoghue and Rabin, 1999a, 1999b). An agent is partially naive if the self in every period $t$ underestimates the present-bias of her future selves, believing that her future selves' present bias is $\tilde{\beta} \in(\beta, 1)$; in the extreme, if the present self believes that her future selves are time-consistent, i.e. $\tilde{\beta}=1$, she is said to be completely naive. On the other hand, an agent is sophisticated if the self in every period $t$ correctly knows her future selves' present-bias $\beta$ and anticipates their behavior when making her period- $t$ decision, i.e., if $\tilde{\beta}=\beta$.

Following previous studies of time-inconsistent preferences, we will analyze the behavior of an agent by thinking of the single individual as consisting of many autonomous selves, one for each period. Each period- $t$ self chooses her current behavior to maximize her current utility $U_{t}\left(u_{t}, u_{t+1}, \ldots\right)$, while her future selves control her subsequent decisions.

More specifically, let the observable state variable in period $t$ be $x_{t} \in \mathcal{X}$ where $\mathcal{X}$ denotes the support of the state variables and the unobservable choice-specific shock $\varepsilon_{i t} \in R$, and $\varepsilon_{t}=$ $\left(\varepsilon_{1 t}, \ldots, \varepsilon_{I t}\right) \in R^{I}$. A strategy profile for all selves is $\sigma \equiv\left\{\sigma_{t}\right\}_{t=1}^{\infty}$ where $\sigma_{t}: \mathcal{X} \times R^{I} \rightarrow \mathcal{I}$ for all $t$. It specifies for each self her action in all possible states and under all possible realizations of shock vectors. For any strategy profile $\boldsymbol{\sigma}$, write $\boldsymbol{\sigma}_{t}^{+} \equiv\left\{\sigma_{k}\right\}_{k=t}^{\infty}$ as the continuation strategy profile from period $t$ on.

To define and characterize the equilibrium of the intra-personal game of an agent with potentially time-inconsistent preferences, we first write $V_{t}\left(x_{t}, \varepsilon_{t} ; \boldsymbol{\sigma}_{t}^{+}\right)$as the agent's period- $t$ expected continuation utility when the state variable is $x_{t}$ and the shock vector is $\varepsilon_{t}$ under her long-run time preference for a given continuation strategy profile $\boldsymbol{\sigma}_{t}^{+}$. We can think of $V_{t}\left(x_{t}, \varepsilon_{t} ; \boldsymbol{\sigma}_{t}^{+}\right)$as representing (hypothetically) her intertemporal preferences from some prior perspective when her own present-bias is irrelevant. Specifically, $V_{t}\left(x_{t}, \varepsilon_{t} ; \boldsymbol{\sigma}_{t}^{+}\right)$must satisfy:

$$
V_{t}\left(x_{t}, \boldsymbol{\varepsilon}_{t} ; \boldsymbol{\sigma}_{t}^{+}\right)=u_{\sigma_{t}\left(x_{t}, \varepsilon_{t}\right)}^{*}\left(x_{t}, \varepsilon_{\sigma_{t}\left(x_{t}, \varepsilon_{t}\right) t}\right)+\delta \mathrm{E}\left[V_{t+1}\left(x_{t+1}, \boldsymbol{\varepsilon}_{t+1} ; \boldsymbol{\sigma}_{t+1}^{+}\right) \mid x_{t}, \sigma_{t}\left(x_{t}, \boldsymbol{\varepsilon}_{t}\right)\right]
$$

where $\sigma_{t}\left(x_{t}, \varepsilon_{t}\right) \in \mathcal{I}$ is the choice specified by strategy $\sigma_{t}$, and the expectation is taken over both the future state $x_{t+1}$ and $\varepsilon_{t+1}$.

We will define the equilibrium for a partially naive agent whose period- $t$ self believes that, 
beginning next period, her future selves will behave optimally with a present-bias factor of $\tilde{\beta} \in$ $[\beta, 1]$. Following O'Donoghue and Rabin (1999b, 2001), we first define the concept of an agent's perceived continuation strategy profile by her future selves.

Definition 2. The perceived continuation strategy profile for a partially naive agent is a strategy profile $\tilde{\boldsymbol{\sigma}} \equiv\left\{\tilde{\sigma}_{t}\right\}_{t=1}^{\infty}$ such that for all $t=1,2, \ldots$, all $x_{t} \in \mathcal{X}$, and all $\varepsilon_{t} \in R^{I}$,

$$
\tilde{\sigma}_{t}\left(x_{t}, \boldsymbol{\varepsilon}_{t}\right)=\arg \max _{i \in \mathcal{I}}\left\{u_{i}^{*}\left(x_{t}, \epsilon_{i t}\right)+\tilde{\beta} \delta \mathrm{E}\left[V_{t+1}\left(x_{t+1}, \varepsilon_{t+1} ; \tilde{\boldsymbol{\sigma}}_{t+1}^{+}\right) \mid x_{t}, i\right]\right\} .
$$

That is, if an agent is partially naive with perceived present-bias by future selves at $\tilde{\beta}$, then her period- $t$ self will anticipate that her future selves will follow strategies $\tilde{\boldsymbol{\sigma}}_{t+1}^{+} \equiv\left\{\tilde{\sigma}_{k}\right\}_{k=t+1}^{\infty}$. Note, importantly, what the strategy profile $\tilde{\boldsymbol{\sigma}} \equiv\left\{\tilde{\sigma}_{t}\right\}_{t=1}^{\infty}$ describes is the perception of the partially naive agent regarding what her future selves will play. It is not what will generate the actual play that we observe in the data. What we actually observe is generated from the perception-perfect strategy profile that we now define.

Definition 3. A perception-perfect strategy profile for a partially naive agent is a strategy profile $\sigma^{*} \equiv$ $\left\{\sigma_{t}^{*}\right\}_{t=1}^{\infty}$ such that, for all $t=1,2, \ldots$, all $x_{t} \in \mathcal{X}$, and all $\varepsilon_{t} \in R^{I}$,

$$
\sigma_{t}^{*}\left(x_{t}, \boldsymbol{\varepsilon}_{t}\right)=\arg \max _{i \in \mathcal{I}}\left\{u_{i}^{*}\left(x_{t}, \varepsilon_{i t}\right)+\beta \delta \mathrm{E}\left[V_{t+1}\left(x_{t+1}, \boldsymbol{\varepsilon}_{t+1} ; \tilde{\boldsymbol{\sigma}}_{t+1}^{+}\right) \mid x_{t}, i\right]\right\} .
$$

That is, $\boldsymbol{\sigma}^{*}$ is the best response of the current self with $(\beta, \delta)$-preference against $\tilde{\boldsymbol{\sigma}}$, the perceived continuation strategy profile of her future selves. It is key to note the difference and connection between $\tilde{\boldsymbol{\sigma}}$ and $\boldsymbol{\sigma}^{*} . \tilde{\boldsymbol{\sigma}}$ is the unobserved perception of the partially naive agent regarding what her future selves will do, under the partial naivety assumption that her future selves do not suffer from the present bias as described by the parameter $\beta$, but instead is governed by present bias parameter $\tilde{\beta}$ that may differ from $\beta . \sigma^{*}$ is what the self in each period will optimally choose to do, and the actions generated from $\sigma^{*}$ are what will be observed in the data. Note also that when $\beta$ and $\tilde{\beta}$ coincide, i.e., when the agent is sophisticated, we have $\boldsymbol{\sigma}^{*}=\tilde{\boldsymbol{\sigma}}$.

Assumption 2. (Stationarity) We assume that the observed choices are generated under the stationary perception-perfect strategy profile of the infinite horizon dynamic game played among different selves of the decision makers. 


\subsection{Decision Process}

Now we describe the decision process of the decision maker. First, define the deterministic component of the current choice-specific value function, $W_{i}(x)$, as follows:

$$
W_{i}(x)=u_{i}(x)+\beta \delta \sum_{x^{\prime} \in \mathcal{X}} V\left(x^{\prime}\right) \pi\left(x^{\prime} \mid x, i\right),
$$

where $\pi\left(x^{\prime} \mid x, i\right)$ denotes the transition probabilities for state variables $x$ when action $i$ is taken; and $V(\cdot)$ is the perceived long-run value function defined as:

$$
V(x) \equiv \mathrm{E}_{\boldsymbol{\varepsilon}} V(x, \boldsymbol{\varepsilon} ; \tilde{\boldsymbol{\sigma}})
$$

where $V(x, \varepsilon ; \tilde{\boldsymbol{\sigma}})$ is the stationary value function defined according to (2) under the perceived continuation strategy profile $\tilde{\boldsymbol{\sigma}}$ for a partially naive agent as defined in Definition 2.

Using $V(\cdot)$ as defined in (4), we can also define the choice-specific value function of the next-period self as perceived by the current self, $Z_{i}(x)$, as follows:

$$
Z_{i}(x)=u_{i}(x)+\tilde{\beta} \delta \sum_{x^{\prime} \in \mathcal{X}} V\left(x^{\prime}\right) \pi\left(x^{\prime} \mid x, i\right)
$$

There are two key difference between $W_{i}(x)$ and $Z_{i}(x)$. The first difference is in how they discount the future streams of payoffs: in $W_{i}(x)$ the payoff $t$ periods removed from the current period is discounted by $\beta \delta^{t}$, while in $Z_{i}(x)$ the payoff $t$ periods removed from now is discounted by $\tilde{\beta} \delta^{t}$. The second difference is interpretational: $W_{i}(x)$ represents how the current-period self evaluates the deterministic component of the payoff from choosing alternative $i$, while $Z_{i}(x)$ is how the current-period self perceives how her next-period self would evaluate the deterministic component of the payoff from choosing alternative $i$. It is obvious but important to note that $W_{i}(x)$ will regulate the current self's optimal choice, but $Z_{i}(x)$ will regulate the perception of the current self regarding the choices of her future selves.

Given $Z_{i}(x)$, we know that the current self's perception of her future self's choice, i.e., $\tilde{\sigma}$ as defined in Definition 2 is simply

$$
\begin{aligned}
\tilde{\sigma}(x, \varepsilon) & =\max _{i \in \mathcal{I}}\left[u_{i}(x)+\varepsilon_{i}+\tilde{\beta} \delta \sum_{x^{\prime} \in \mathcal{X}} V\left(x^{\prime}\right) \pi\left(x^{\prime} \mid x, i\right)\right] \\
& =\max _{i \in \mathcal{I}}\left[Z_{i}(x)+\varepsilon_{i}\right] .
\end{aligned}
$$

Let us define the probability of choosing alternative $j$ by the the next period self as perceived by 
the current period self, $\tilde{P}_{j}(x)$, when the next-period's state is $x$ :

$$
\begin{aligned}
\tilde{P}_{j}(x) & =\operatorname{Pr}[\tilde{\sigma}(x, \varepsilon)=j] \\
& =\operatorname{Pr}\left[Z_{j}(x)+\varepsilon_{j} \geq Z_{j^{\prime}}+\varepsilon_{j^{\prime}} \text { for all } j^{\prime} \neq j\right] .
\end{aligned}
$$

With the characterization of $\tilde{\sigma}(x, \varepsilon)$, we can now provide a characterization of $V(\cdot)$. For this purpose, further denote the perceived choice-specific long-run value function $V_{i}(x)$ as follows:

$$
V_{i}(x)=u_{i}(x)+\delta \sum_{x^{\prime} \in \mathcal{X}} V\left(x^{\prime}\right) \pi\left(x^{\prime} \mid x, i\right)
$$

According to the definition of $V(\cdot)$ as given by (4), $V(x)$ is simply the expected value of $V_{i}(x)+\varepsilon_{i}$, where $i$ is the chosen alternative according to $\tilde{\sigma}(x, \varepsilon)$; that is, we have the following relationship:

$$
V(x)=\mathrm{E}_{\varepsilon}\left[V_{\tilde{\sigma}(x, \varepsilon)}(x)+\varepsilon_{\tilde{\sigma}(x, \varepsilon)}\right] .
$$

Now note from (5) and (8), we have

$$
V_{i}(x)=Z_{i}(x)+(1-\tilde{\beta}) \delta \sum_{x^{\prime} \in \mathcal{X}} V\left(x^{\prime}\right) \pi\left(x^{\prime} \mid x, i\right) .
$$

Relationship (10) is crucial as it allows us to rewrite (9) as:

$$
\begin{aligned}
V(x) & =\mathrm{E}_{\varepsilon}\left[V_{\tilde{\sigma}(x, \varepsilon)}(x)+\varepsilon_{\tilde{\sigma}(x, \varepsilon)}\right. \\
& =\mathrm{E}_{\varepsilon}\left[Z_{\tilde{\sigma}(x, \boldsymbol{\varepsilon})}(x)+\varepsilon_{\tilde{\sigma}(x, \varepsilon)}+(1-\tilde{\beta}) \delta \sum_{x^{\prime} \in \mathcal{X}} V\left(x^{\prime}\right) \pi\left(x^{\prime} \mid x, \tilde{\sigma}(x, \varepsilon)\right)\right] \\
& =\mathrm{E}_{\varepsilon} \max _{i \in \mathcal{I}}\left[Z_{i}(x)+\varepsilon_{i}\right]+(1-\tilde{\beta}) \delta \mathrm{E}_{\varepsilon} \sum_{x^{\prime} \in \mathcal{X}} V\left(x^{\prime}\right) \pi\left(x^{\prime} \mid x, \tilde{\sigma}(x, \varepsilon)\right) \\
& =\mathrm{E}_{\varepsilon} \max _{i \in \mathcal{I}}\left[Z_{i}(x)+\varepsilon_{i}\right]+(1-\tilde{\beta}) \delta \sum_{j \in \mathcal{I}}\left[\tilde{P}_{j}(x) \sum_{x^{\prime} \in \mathcal{X}} V\left(x^{\prime}\right) \pi\left(x^{\prime} \mid x, j\right)\right]
\end{aligned}
$$

where the second equality just follows from (10); and the third equality follows from (6) and thus

$$
\mathrm{E}_{\boldsymbol{\varepsilon}}\left[Z_{\tilde{\sigma}(x, \boldsymbol{\varepsilon})}(x)+\varepsilon_{\tilde{\sigma}(x, \varepsilon)}\right]=\mathrm{E}_{\varepsilon} \max _{i \in \mathcal{I}}\left[Z_{i}(x)+\varepsilon_{i}\right]
$$


and the fourth equality follows from the fact that

$$
\begin{aligned}
\mathrm{E}_{\varepsilon} \sum_{x^{\prime} \in \mathcal{X}} V\left(x^{\prime}\right) \pi\left(x^{\prime} \mid x, \tilde{\sigma}(x, \boldsymbol{\varepsilon})\right) & =\sum_{j \in \mathcal{I}}\left[\operatorname{Pr}(\tilde{\sigma}(x, \boldsymbol{\varepsilon})=j) \sum_{x^{\prime} \in \mathcal{X}} V\left(x^{\prime}\right) \pi\left(x^{\prime} \mid x, j\right)\right] \\
& =\sum_{j \in \mathcal{I}}\left[\tilde{P}_{j}(x) \sum_{x^{\prime} \in \mathcal{X}} V\left(x^{\prime}\right) \pi\left(x^{\prime} \mid x, j\right]\right) .
\end{aligned}
$$

Now we make two additional standard assumptions about the transition of the state variables and the distribution of the shocks [see, e.g., Rust (1994b)]:

Assumption 3. (Conditional Independence):

$$
\begin{aligned}
& \pi\left(x_{t+1}, \varepsilon_{t+1} \mid x_{t}, \varepsilon_{t}, d_{t}\right)=q\left(\varepsilon_{t+1} \mid x_{t+1}\right) \pi\left(x_{t+1} \mid x_{t}, d_{t}\right) \\
& q\left(\varepsilon_{t+1} \mid x_{t+1}\right)=q(\varepsilon)
\end{aligned}
$$

Assumption 4. (Extreme Value Distribution): $\varepsilon_{t}$ is i.i.d extreme value distributed.

Remark 1. It is well-known that the distribution of the choice-specific shocks to payoffs in discrete choice models are not non-parametrically identified (see Magnac and Thesmar 2002, for example). Thus one has to make an assumption about the distribution of $\varepsilon$. We make the extreme value distribution assumption for simplicity, but it could be replaced by any other distribution $G$.

With the above preliminary notations, now we can describe how the agent will make the choices when the state variables are given by $(x, \varepsilon)$. From Definition 3 for perception perfect strategy profile and Equation (3), we know that the current period decision maker will choose $i$ if and only if

$$
i \in \arg \max _{i \in \mathcal{I}}\left\{W_{i}(x)+\varepsilon_{i}\right\} .
$$

That is, the perception-perfect strategy profile $\sigma^{*}(x, \varepsilon)$ is:

$$
\sigma^{*}(x, \varepsilon)=\arg \max _{i \in \mathcal{I}}\left\{W_{i}(x)+\varepsilon_{i}\right\}
$$

Under Assumption 4, the probability of observing action $i$ being chosen at a given state variable $x$ is:

$$
P_{i}(x)=\operatorname{Pr}\left[W_{i}(x)+\varepsilon_{i}>\max _{j \in \mathcal{I} \backslash\{i\}}\left\{W_{j}(x)+\varepsilon_{j}\right\}\right]=\frac{\exp \left[W_{i}(x)\right]}{\sum_{j=0}^{I} \exp \left[W_{j}(x)\right]} .
$$

$P_{i}(x)$ is the current-period self's equilibrium choice probabilities and will be observed in the data.

Now we derive some important relationships that will be used in our identification exercise 
below. First, note that by combining (3) and (5), we have that:

$$
Z_{i}(x)-u_{i}(x)=\frac{\tilde{\beta}}{\beta}\left[W_{i}(x)-u_{i}(x)\right]
$$

Since both $Z_{i}(\cdot)$ and $W_{i}(\cdot)$ depends on $V(\cdot)$, we would like to use (11) to derive a characterization of $V(\cdot)$. Note that under Assumptions 4, we have:

$$
\mathrm{E}_{\varepsilon} \max _{i \in \mathcal{I}}\left\{Z_{i}(x)+\varepsilon_{i}\right\}=\ln \left\{\sum_{i \in \mathcal{I}} \exp \left[Z_{i}(x)\right]\right\} .
$$

Moreover, from (7), we have that

$$
\tilde{P}_{j}(x)=\frac{\exp \left[Z_{j}(x)\right]}{\sum_{i=0}^{I} \exp \left[Z_{i}(x)\right]}
$$

Using (14) and (15), we can rewrite (11) as

$$
V(x)=\ln \left\{\sum_{i \in \mathcal{I}} \exp \left[Z_{i}(x)\right]\right\}+(1-\tilde{\beta}) \delta \sum_{j \in \mathcal{I}} \frac{\exp \left[Z_{j}(x)\right]}{\sum_{i=0}^{I} \exp \left[Z_{i}(x)\right]} \sum_{x^{\prime} \in \mathcal{X}} V\left(x^{\prime}\right) \pi\left(x^{\prime} \mid x, j\right) .
$$

The three set of equations (5), (13) and (16) will form the basis of our identification argument below. Let us first make a few useful remarks.

Remark 2. We have three value functions $\left\{W_{i}(x), Z_{i}(x), V_{i}(x): x \in \mathcal{X}\right\}$ as defined respectively in (3), (5) and (8). Both $W_{i}(\cdot)$ and $Z_{i}(\cdot)$ are related to $V_{i}(\cdot)$. It is worth emphasizing that $W_{i}(x)$ will regulate the current self's choice behavior as demonstrated by (12); and $Z_{i}(x)$ will regulate the current self's perception of future selves choices as demonstrated by (15). $V_{i}(x)$ is an auxiliary value function that simply uses the long-run discount factor $\delta$ to evaluate the payoffs from the choices that the current self perceives that will be made by her future selves.

Remark 3. If $\tilde{\beta}=1$, i.e., if the decision maker is completely naive, we can see from (10) that $V_{i}(x)=Z_{i}(x)$ for all $x$. This makes sense because when $\tilde{\beta}=1$, the current self perceives her future selves to be time consistent. Thus the current self is already perceiving her future selves to be behaving according to the long run discount factor $\delta$ only.

Remark 4. If $\tilde{\beta}=\beta$, i.e., when an agent is sophisticated, then Eqs. (3) and (5) tell us that $W_{i}(x)=Z_{i}(x)$. That is, if the decision maker is sophisticated, then the current self's own choice rule will be identical to what she perceives to be her future self's choice rule.

Remark 5. When the decision maker is partially naive, there are two distinct value functions $W_{i}(x)$ and $Z_{i}(x)$ that separately regulate the choice of the current self and the perceived choice of her future selves. 


\begin{tabular}{|c|c|c|}
\hline Notation & Interpretation & Equation/Definition \\
\hline$u_{i}(x)$ & $\begin{array}{l}\text { Deterministic payoff from choosing alternative } i \\
\text { when state vector is } x\end{array}$ & \\
\hline$u_{i}^{*}(x)$ & $\begin{array}{l}\text { Payoff, including the choice-specific shock, from choosing } \\
\text { alternative } i \text { when state vector is } x: u_{i}^{*}(x) \equiv u_{i}(x)+\varepsilon_{i}\end{array}$ & Eq. (1) \\
\hline$\beta$ & The present-bias factor & Definition (1) \\
\hline$\tilde{\beta}$ & The partial naivety parameter: $\tilde{\beta} \in[\beta, 1]$ & \\
\hline$\delta$ & The standard discount factor & Definition (1) \\
\hline$V_{i}(x)$ & Perceived choice-specific long-run value function & Eq. (8) \\
\hline$V(x)$ & Perceived long-run value function & Eq. (4) \\
\hline$W_{i}(x)$ & Current choice-specific value function & Eq. (3) \\
\hline$Z_{i}(x)$ & $\begin{array}{l}\text { Choice-specific value function of the next-period self } \\
\text { as perceived by the current self }\end{array}$ & Eq. (5) \\
\hline$\tilde{\sigma}(x, \varepsilon)$ & Perceived continuation strategy profile for a partially naive agent & Definition (2) \\
\hline$\sigma^{*}(x, \varepsilon)$ & Perception-perfect strategy profile for a partially naive agent & Definition (3) \\
\hline
\end{tabular}

Table 1: Summary of Key Notations.

Equation (13) clarifies that it is the fact that we allow for potential naivety in the hyperbolic model that is creating the wedge between $W_{i}(x)$ and $Z_{i}(x)$ : if $\tilde{\beta}=\beta$, i.e., if agents are sophisticated (even when they suffer from present bias), it would be true that $W_{i}(x)=Z_{i}(x)$. This is an important point because, as we see in (12), the observed choice probabilities (our data) would provide direct information about $W_{i}(x)$, without needing any information about the discount factors. Thus when $\tilde{\beta}=\beta$, the observed choice probabilities also provide direct information about $Z_{i}(x)$; but when $\tilde{\beta}$ and $\beta$ are potentially not equal, we can no longer learn about $Z_{i}(x)$ directly from the observed choice probabilities.

Summary. Table 1 summarizes the main notations we have introduced up to now.

\subsection{Relationship with the Dynamic Games Literature}

We analyze the observed outcome of the dynamic discrete choice problem of a hyperbolic discounting decision process as the equilibrium outcome of an intra-personal game with the players being the selves at different periods. Thus our paper represents an interesting intermediate case between the classic literature on estimating single-agent dynamic discrete choice decision problems and the more recent literature on estimating dynamic games. It is worth pointing out that there are two crucial differences between the intra-personal games we analyze for agents with time- 
inconsistent time preferences and those in the existing dynamic games literature.

The first key difference is that in our case, we do not observe the actions of all the players. More specifically, the outcomes - choices and the evolutions of the state variables - we observe in the data are affected only by the current selves, even though the current selves' choices are impacted by their perception of future selves' actions. The current self's perception of how her future selves will play has to be inferred by the researcher using the equilibrium restriction imposed by the theory. As can be seen from the above discussion, $\tilde{P}_{j}(x)$ as defined in (15), captures the current self's perception of how her future selves will play, which is crucial for us to understand the current self's actual choices. However, as a researcher, we do not observe $\tilde{P}_{j}$, only observe $P_{j}$, the choice probabilities by the current self. In the standard dynamic games literature, it is always assumed that the action of all the players are observed.

Secondly, the dynamic games literature (e.g. Bajari, Benkard and Levin 2007) may allow for players to have different contemporaneous payoff functions, in our setting, however, the payoffs for the players - the current self and the future selves - differ only in their time preferences; moreover, under hyperbolic discounting, we are assuming a rather restricted form of difference in time preferences between the selves in different periods.

\section{Identification}

\subsection{Data and Preliminaries}

Before we describe our results on identification, let us assume that we have access to a data set that provides us with the following information:

\section{DATA:}

- (Conditional Choice Probabilities) For all $x \in \mathcal{X}$, we observe the choice probabilities $P_{i}(x)$ for all $i \in \mathcal{I}$;

- (Transitional Probabilities for Observable State Variables) For all $\left(x, x^{\prime}\right) \in \mathcal{X}^{2}$, all $i \in \mathcal{I}$, we observe the transition probabilities $\pi\left(x^{\prime} \mid x, i\right)$; we denote

$$
\boldsymbol{\pi} \equiv\left\{\pi\left(x^{\prime} \mid x, i\right):\left(x, x^{\prime}\right) \in \mathcal{X}^{2}, i \in \mathcal{I}\right\}
$$

- (Short Panels) We have access to at least two periods of the above data, even though the data results from a stationary infinite horizon model. 
Following Magnac and Thesmar (2002), we assume that the structure of the model, denoted by $\theta$, is defined by the following parameters: ${ }^{9}$

$$
\theta=\left\{(\beta, \tilde{\beta}, \delta), G,\left\langle\left\{u_{i}(x), Z_{i}\left(x^{\prime}\right), V_{i}\left(x^{\prime}\right): i \in \mathcal{I}, x \in \mathcal{X}, x^{\prime} \in \mathcal{X}\right\}\right\rangle\right\}
$$

where $G(\cdot)$, the distribution of the choice-specific payoff shocks $\varepsilon_{i t}$, is assumed to have a Type-I extreme value distribution by Assumption 4. Note that the elements in $\theta$ in our setting differs from those in Magnac and Thesmar (2002) in that we have two additional parameters $\beta$ and $\tilde{\beta}$ that measure present bias and naivety; moreover, the interpretation of $V_{i}\left(x^{\prime}\right)$ in our paper differs from theirs. In their paper $V_{i}\left(x^{\prime}\right)$ directly informs about the actual choice probabilities of the decision maker in the second period because $\operatorname{Pr}\left(i \mid x^{\prime}\right)=\operatorname{Pr}\left(V_{i}\left(x^{\prime}\right)+\varepsilon_{i}>V_{j}\left(x^{\prime}\right)+\varepsilon_{j} \forall j \neq i\right)$. In our paper, $Z_{i}\left(x^{\prime}\right)$ captures the current self's perception of the choice probability of the next period's self, which is never actually observed in the data; while $V_{i}\left(x^{\prime}\right)$ is just an auxiliary value function to account for the exponentially discounted payoff streams from the perceived choices made according to $\tilde{\sigma}(x, \varepsilon)$. Another difference is that in Magnac and Thesmar (2002), the vector $\left\{V_{i}\left(x^{\prime}\right): x^{\prime} \in \mathcal{X}\right\}$ are completely free parameters; in our setting, however, neither $Z_{i}\left(x^{\prime}\right)$ and $V_{i}\left(x^{\prime}\right)$ are completely free parameters as they are subject to the restriction that they have to satisfy (5), (13) and (16).

We denote by $\Theta$ the set of all permissible structures. The set $\Theta$ requires that the structure satisfies the assumptions we adopted in the model, as well as the restrictions (5), (13) and (16).

Given any structure $\theta \in \Theta$, the model predicts the probability that an agent will choose alternative $i \in \mathcal{I}$ in state $x \in \mathcal{X}$, which we denote by $\hat{P}_{i}(x ; \theta)$ and is given by

$$
\hat{P}_{i}(x ; \theta)=\operatorname{Pr}\left\{u_{i}(x)+\varepsilon_{i}+\beta \delta \sum_{x^{\prime} \in \mathcal{X}} V\left(x^{\prime}\right) \pi\left(x^{\prime} \mid x, i\right)=\max _{j \in \mathcal{I}}\left[u_{j}(x)+\varepsilon_{j}+\beta \delta \sum_{x^{\prime} \in \mathcal{X}} V\left(x^{\prime}\right) \pi\left(x^{\prime} \mid x, j\right)\right] \mid x, \theta\right\} .
$$

As is standard in the identification literature, we call the predicted choice probabilities $\hat{P}_{i}(x ; \theta)$ as the reduced form of structure $\theta \in \Theta$. We say that two structures $\theta, \theta^{\prime} \in \Theta$ are observationally equivalent if

$$
\hat{P}_{i}(x ; \theta)=\hat{P}_{i}\left(x ; \theta^{\prime}\right) \forall i \in \mathcal{I} \text { and } x \in \mathcal{X} .
$$

A model is said to be identified if and only if for any $\theta, \theta^{\prime} \in \Theta, \theta=\theta^{\prime}$ if they are observationally equivalent.

\footnotetext{
${ }^{9}$ We could have included the vector $\left\{W_{i}(x): i \in \mathcal{I}, x \in \mathcal{X}\right\}$ as part of the model parameters as well. But as seen from Eq. (17), $W_{i}(x)$ can be straightforwardly inferred from the data of choice probabilities $P_{i}(x)$, subject to a normalization. For this reason, we exclude them from our list of model parameters.
} 


\subsection{Identification Results}

We first describe identification of $\left\langle\left\{u_{i}(x), Z_{i}\left(x^{\prime}\right), V_{i}\left(x^{\prime}\right): i \in \mathcal{I}, x \in \mathcal{X}, x^{\prime} \in \mathcal{X}\right\}\right\rangle$ with for a given set of discount factors $\langle\beta, \tilde{\beta}, \delta\rangle$. Then we provide conditions pertinent to the identification of $\langle\beta, \tilde{\beta}, \delta\rangle$.

3.2.1 Identification of $\left\langle\left\{u_{i}(x), Z_{i}\left(x^{\prime}\right), V_{i}\left(x^{\prime}\right): i \in \mathcal{I}, x \in \mathcal{X}, x^{\prime} \in \mathcal{X}\right\}\right\rangle$ for Given $\langle\beta, \tilde{\beta}, \delta\rangle$

For any given joint distribution $G$ of $\tilde{\varepsilon} \equiv\left(\varepsilon_{1}, \ldots, \varepsilon_{I}\right)$, the choice probability vector $\mathbf{P}(x)=$ $\left(P_{1}(x), \ldots, P_{I}(x)\right)$ is a mapping $Q$ of $\mathbf{W}(x)=\left(W_{0}(x), W_{1}(x), \ldots, W_{I}(x)\right)$. Hotz and Miller (1993) showed that the mapping $Q$ can be inverted if for each $i \in \mathcal{I}$, one of the $W_{i}(x)$ is normalized. That is, one can find

$$
D_{i}(x) \equiv W_{i}(x)-W_{0}(x)=Q_{i}(\mathbf{P}(x) ; G)
$$

where $Q_{i}$ is the $i^{\text {th }}$ component of the inverse $Q$. Under our Assumption 4 (that $\varepsilon_{i}$ has i.i.d Type-I Extreme Value distribution), the mapping $Q_{i}$ is especially simple [following from (12)]:

$$
D_{i}(x)=W_{i}(x)-W_{0}(x)=\ln \frac{P_{i}(x)}{P_{0}(x)}
$$

Since we observe $P_{i}(x)$ and $P_{0}(x)$ from the data, we immediately learn about $D_{i}(x)$. We thus proceed as if $D_{i}(x)$ is observable.

From Eq. (13), we have that, for all $i \in \mathcal{I}$,

$$
Z_{i}(x)=\frac{\tilde{\beta}}{\beta} W_{i}(x)+\left(1-\frac{\tilde{\beta}}{\beta}\right) u_{i}(x)
$$

Together with (17), we have, for all $i \in \mathcal{I} \backslash\{0\}$ and $x \in \mathcal{X}$,

$$
Z_{i}(x)-Z_{0}(x)=\frac{\tilde{\beta}}{\beta} D_{i}(x)+\left(1-\frac{\tilde{\beta}}{\beta}\right)\left[u_{i}(x)-u_{0}(x)\right] .
$$

Now let us examine the system of equations defined by Eq. (16), which can be re-written, using (15), as, for all $x \in \mathcal{X}$,

$$
V(x)=Z_{0}(x)+\ln \left\{\sum_{i \in \mathcal{I}} \exp \left[Z_{i}(x)-Z_{0}(x)\right]\right\}+(1-\tilde{\beta}) \delta \sum_{j \in \mathcal{I}} \tilde{P}_{j}(x) \sum_{x^{\prime} \in \mathcal{X}} V\left(x^{\prime}\right) \pi\left(x^{\prime} \mid x, j\right) .
$$

Note that if we take $\left\{\beta, \tilde{\beta}, \delta,\left\langle Z_{i}(x): x \in \mathcal{X}\right\rangle\right\}$ as given, Eq. (20) is just a system of $X$ linear equa- 
tion in $X$ unknowns, namely, $\mathbf{V} \equiv[V(1), \ldots, V(X)]^{T} \cdot 10$

Specifically, if we stack $\left[Z_{0}(x)+\ln \left\{\sum_{i \in \mathcal{I}} \exp \left[Z_{i}(x)-Z_{0}(x)\right]\right\}\right]_{x \in \mathcal{X}}$ into an $X \times 1$ column vector $\mathbf{A}$, denote by $\tilde{\mathbf{P}} \equiv\left[\begin{array}{lll}\tilde{\mathbf{P}}_{0} & \cdots & \tilde{\mathbf{P}}_{I}\end{array}\right]$ the $X \times[(I+1) X]$ matrix of choice probabilities, ${ }^{11}$ and properly stack up the transition matrices $\pi\left(x^{\prime} \mid x, j\right)$ into an $[(I+1) X] \times X$ matrix as: ${ }^{12}$

$$
\Pi \equiv\left[\begin{array}{c}
\Pi_{0} \\
\vdots \\
\Pi_{I}
\end{array}\right]
$$

we can write (20) as

$$
\mathbf{V}=\mathbf{A}+(1-\tilde{\beta}) \delta \tilde{\mathbf{P}} \mathbf{\Pi V}
$$

which yields the solution of $\mathbf{V}$ as a function of $\left\{\beta, \tilde{\beta}, \delta,\left\langle Z_{i}(x): x \in \mathcal{X}\right\rangle\right\}$ to be:

$$
\mathbf{V}=[\mathbf{I}-(1-\tilde{\beta}) \delta \tilde{\mathbf{P}} \boldsymbol{\Pi}]^{-1} \mathbf{A}
$$

Thus for fixed values of $(\beta, \tilde{\beta}, \delta)$, we can plug (21) into (5) and obtain, for all $x \in \mathcal{X}$, and all $i \in \mathcal{I} \equiv\{0,1, \ldots, I\}$,

$$
\begin{aligned}
Z_{i}(x) & =u_{i}(x)+\tilde{\beta} \delta \sum_{x^{\prime} \in \mathcal{X}} V\left(x^{\prime}\right) \pi\left(x^{\prime} \mid x, i\right) \\
& =u_{i}(x)+\tilde{\beta} \delta \boldsymbol{\Pi}_{i}(x) \mathbf{V} \\
& =u_{i}(x)+\tilde{\beta} \delta \boldsymbol{\Pi}_{i}(x)[\mathbf{I}-(1-\tilde{\beta}) \delta \tilde{\mathbf{P}} \boldsymbol{\Pi}]^{-1} \mathbf{A},
\end{aligned}
$$

where $\Pi_{i}(x)=[\pi(1 \mid x, i), \ldots, \pi(X \mid x, i)]$ is an $X \times 1$ vector as defined in footnote 12 .

\footnotetext{
${ }^{10}$ Note that all the terms $\tilde{P}_{j}(x)$ just depend on $Z_{i}(\cdot)$.

${ }^{11}$ For each $j \in \mathcal{I}, \tilde{\mathbf{P}}_{j}$ is the $X \times X$ matrix organized as: $\tilde{\mathbf{P}}_{j}=\left[\begin{array}{cccc}\tilde{P}_{j}(0) & 0 & \cdots & 0 \\ 0 & \tilde{P}_{j}(1) & \cdots & 0 \\ \mathbf{0} & \mathbf{0} & \ddots & \mathbf{0} \\ 0 & 0 & \cdots & \tilde{P}_{j}(X)\end{array}\right]$.
}

${ }^{12}$ Here, $\Pi_{j}$ is an $X \times X$ matrix defined by

$$
\boldsymbol{\Pi}_{j}=\left[\begin{array}{c}
\boldsymbol{\Pi}_{j}(1) \\
\boldsymbol{\Pi}_{j}(2) \\
\vdots \\
\boldsymbol{\Pi}_{j}(X)
\end{array}\right]=\left[\begin{array}{ccc}
\pi(1 \mid 1, j) & \ldots & \pi(X \mid 1, j) \\
\pi(1 \mid 2, j) & \ldots & \pi(X \mid 2, j) \\
& \vdots & \\
\pi(1 \mid X, j) & \ldots & \pi(X \mid X, j)
\end{array}\right]
$$


Now consider the system of equations given by (19) and (22). We know from the standard theories of discrete choice that we have to normalize the utility for the reference alternative 0 , which without loss of generality we set $u_{0}(x)=0$ for all $x \in \mathcal{X}$. Thus, the unknowns contained in the equation system of (19) and (22) include the following unknowns: $(I+1) \times X$ values for $\left\{Z_{i}(x): i \in \mathcal{I}, x \in \mathcal{X}\right\}$ and $I \times X$ values for $\left\{u_{i}(x): i \in \mathcal{I} /\{0\}, x \in \mathcal{X}\right\}$. That is, the total number of unknowns is $(2 I+1) \times X$. It is also easy to see that the total number of equations in the system is also equal to $(2 I+1) \times X: I \times X$ equations in (19) and $(I+1) \times X$ equations in (22).

Of course, having the same number of equations as the number of unknowns does not guarantee the existence, or the uniqueness of the solutions. We will describe how the number of solutions affect our estimation procedure below.

Note that in the equation system of (19) and (22), the three discounting parameters $(\beta, \tilde{\beta}, \delta)$ appear in three different combinations: first, in Eqs. (22), both $\tilde{\beta} \delta$ and $(1-\tilde{\beta}) \delta$ appear; second, $\tilde{\beta} / \beta$ appears in Eqs. (19). The fact that $\tilde{\beta} \delta,(1-\tilde{\beta}) \delta$ and $\tilde{\beta} / \beta$ appear in the equation system is crucial for the separate estimation of the $(\beta, \tilde{\beta}, \delta)$ parameters. ${ }^{13}$ It is important to emphasize that the non-linearities of how the three parameters $(\beta, \tilde{\beta}, \delta)$ enter our problem is not by ad hoc assumptions, instead they are driven by theoretical considerations.

Finally, as we will see below, the main variables of interest that we would like to solve in the equation system (19) and (22) are the $I \times X$ values for $\left\{u_{i}(x): i \in \mathcal{I} /\{0\}, x \in \mathcal{X}\right\}$, and the values for $\left\{Z_{i}(x): i \in \mathcal{I}, x \in \mathcal{X}\right\}$ are only auxiliary. In our actual estimation, we indeed further eliminate $\left\{Z_{i}(x): i \in \mathcal{I}, x \in \mathcal{X}\right\}$ from the equation system (19) and (22) and only solve for $\left\{u_{i}(x): i \in \mathcal{I} /\{0\}, x \in \mathcal{X}\right\}$.

\subsubsection{Exclusion Restriction to Identify $\langle\beta, \tilde{\beta}, \delta\rangle$}

Now we discuss conditions for identification related to $\langle\beta, \tilde{\beta}, \delta\rangle$. This discussion is closely related to that in Magnac and Thesmar (2002). We impose the following exclusion restriction assumption:

Assumption 5. (Exclusion Restriction) There exist state variable $x_{1} \in \mathcal{X}$ and $x_{2} \in \mathcal{X}$ with $x_{1} \neq x_{2}$, such that

1. for all $i \in \mathcal{I}, u_{i}\left(x_{1}\right)=u_{i}\left(x_{2}\right)$;

\footnotetext{
${ }^{13}$ Note that if the identification of $\langle\tilde{\beta} \delta,(1-\tilde{\beta}) \delta, \tilde{\beta} / \beta\rangle$ is isomorphic to the identifcation of $\langle\beta, \hat{\beta}, \delta\rangle$ :

$$
\delta=\tilde{\beta} \delta+(1-\tilde{\beta}) \delta ; \quad \tilde{\beta}=\frac{\tilde{\beta} \delta}{\tilde{\beta} \delta+(1-\tilde{\beta}) \delta} ; \quad \beta=\frac{\tilde{\beta} \delta}{\tilde{\beta} \delta+(1-\tilde{\beta}) \delta} /\left(\frac{\tilde{\beta}}{\beta}\right) .
$$
}




\section{2. for some $i \in \mathcal{I}, \pi\left(x^{\prime} \mid x_{1}, i\right) \neq \pi\left(x^{\prime} \mid x_{2}, i\right)$.}

More specifically, to satisfy the exclusion restriction assumption, there must exist at least one variable that does not directly affect the contemporaneous utility function $u_{i}(\cdot)$ for all $i \in \mathcal{I}$, but the variable may matter for choices because it affects the transition of state variables. The extent to which individuals' choice probabilities differ at state $x_{1}$ and $x_{2}$ reveals information about the discount factors. This is the key intuition from Magnac and Thesmar's (2002) result where they are interested in identifying a single long-term discount factor $\delta$. In their setting, if $\delta=0$, i.e., if individuals are completely myopic, then the choice probabilities would have been the same under $x_{1}$ and $x_{2}$; to the extent that choice probabilities differ at $x_{1}$ and $x_{2}$, it reveals information about the degree of time discounting. Their intuition, however, can be easily extended to the hyperbolic discounting case, as we will exploit in the proposed estimation strategy below.

For notational simplicity, we will divide the state variables into two groups $\left(x_{r}, x_{e}\right)$ where $x_{r} \in \mathcal{X}_{r}$ refers to the state variables that directly enter the contemporaneous payoff function $u_{i}\left(x_{r}\right)$ and $x_{e} \in \mathcal{X}_{e}$ refers to the state variables that satisfy the exclusion restriction (5). The key idea that the existence of exclusion variables can provide the source of identification of the discount factors is as follows: for any given values for $\langle\beta, \tilde{\beta}, \delta\rangle$, Section 3.2.1 tells us that we can identify, from the observed data, values of $\left\langle\left\{u_{i}(x): i \in \mathcal{I}, x \in \mathcal{X}\right\}\right\rangle$, to the extent that $\langle\beta, \tilde{\beta}, \delta\rangle$ might be consistent with the data at all. However, consider two state vectors $x=\left(x_{r}, x_{e}\right)$ and $x^{\prime}=\left(x_{r}, x_{e}^{\prime}\right)$ that only differ in the exclusion restriction components. If the postulated values of $\langle\beta, \tilde{\beta}, \delta\rangle$ are inconsistent with the true values, there is no guarantee that the identified values of $u_{i}(x)$ and $u_{i}\left(x^{\prime}\right)$ are equal, as required by the exclusion restriction. The exclusion restriction will allow us to select the values of $\langle\beta, \tilde{\beta}, \delta\rangle$ such that the values of $u_{i}(x)$ and $u_{i}\left(x^{\prime}\right)$ identified from the data only differ if $x$ and $x^{\prime}$ differ in components of $x_{r}$.

Before we show how we can use the exclusion restriction to construct estimators for the three discount factors $\langle\beta, \tilde{\beta}, \delta\rangle$, it is useful to provide some intuition as to how $\langle\beta, \tilde{\beta}, \delta\rangle$ come to affect the observed choice behavior by the current self differently.

Distinguishing $\beta$ and $\delta$. It may seem counterintuitive that $\beta$ and $\delta$ could be separately identified in a short two-period panel data set. To provide some intuition, let us consider the case that $\tilde{\beta}=\beta$. The question is: "Can we distinguish the behavior of an agent with exponential discounting rate $\hat{\delta}=\beta \delta$ from the behavior of a sophisticated time-inconsistent agent with preference $(\beta, \delta)$ ?" Under stationarity assumption, if an agent has time consistent exponential discounting rate $\hat{\delta}=\beta \delta$, her expected continuation utilities is completely determined by the observed choice probabilities. To 
see this, observe that in equation (20), if one replaces $\tilde{\beta}$ by 1 and $\delta$ by $\hat{\delta}$, we will have

$$
V(x)=Z_{0}(x)+\ln \left\{\sum_{i \in \mathcal{I}} \exp \left[Z_{i}(x)-Z_{0}(x)\right]\right\},
$$

which only depends on $D_{i}(x)$ when $\beta=\tilde{\beta}$.

However, for a sophisticated time-inconsistent agent with preference $(\beta, \delta)$, there is an incongruence between current self and her perceived future self regarding how they evaluate the future stream of payoffs. Though the current self has to defer to her next-period self in terms of the actual next-period choice that will be chosen, they disagree on how much weight to put on payoffs two-periods from now. It is this incongruence that leads to the last term in Eq. (20), which in turn breaks the tight link between observed choice probabilities and the continuation utilities.

As we demonstrated in Section 3.2.1, the continuation utilities will ultimately determine the identified values of $\left\langle\left\{u_{i}(x): i \in \mathcal{I}, x \in \mathcal{X}\right\}\right\rangle$. Thus $\beta$ and $\delta$ can be distinguished when there are exclusion variables because they requires that $u_{i}(x)$ does not depend on the $x_{e}$ components of the state vector.

Distinguishing $\beta$ and $\tilde{\beta}$. To help provide intuition for why $\beta$ could be distinguished from $\tilde{\beta}$, let us suppose that $\delta=1$. First note that the ratio $\tilde{\beta} / \beta$ appears in term $Z_{i}(x)$ [see Eq. (18)]. This ratio regulates the incongruence between the current self's own behavior and her perception of the behavior of her future selves. Eq. (19) shows that if $\tilde{\beta} / \beta=1$, then $Z_{i}(x)-Z_{0}(x)$ is uniquely determined by the observed $D_{i}(x)$; and thus the current self's perception about her future self's action is identical to her own action. This implies that $\tilde{\beta} / \beta=1$ (and $\delta=1$ ) will pin down completely the identified values of $\left\langle\left\{u_{i}(x): i \in \mathcal{I}, x \in \mathcal{X}\right\}\right\rangle$ from the data, which could be refuted if the identified values of $u_{i}(x)$ do not satisfy the exclusion restrictions, i.e., $u_{i}(x)$ should not depend on the $x_{e}$ components of the state vector.

\subsection{The Exponential Discounting Special Case: $\beta=\tilde{\beta}=1$}

Before discussing our estimation strategy, we here show that the non-linear equation system (19) and (22), for the special case of dynamic discrete choice models with exponential discounting (i.e., the case with $\tilde{\beta}=\beta=1$ ), replicates the known results in the literature. In that case, (19) is reduced to the well-known relationship

$$
Z_{i}(x)-Z_{0}(x)=\ln P_{i}(x)-\ln P_{0}(x)
$$


that is, in standard models the difference in the choice probabilities for alternative $i$ and the reference alternative 0 informs us about the difference in the value from choosing $i$ relative to the value from choosing 0 . This is of course also true when $\tilde{\beta}=\beta<1$. The potential naivety we allow in our setup breaks this direct relationship between $P_{i}(x) / P_{0}(x)$ and $Z_{i}(x)-Z_{0}(x)$.

Moreover, when $\tilde{\beta}=\beta=1$, (22) for $i=0$ is reduced to (using the normalization that $u_{0}(x)=$ $0)$ :

$$
Z_{0}(x)=\delta \sum_{x^{\prime} \in \mathcal{X}} Z_{0}\left(x^{\prime}\right) \pi\left(x^{\prime} \mid x, 0\right)+\delta \sum_{x^{\prime} \in \mathcal{X}} \ln \left[\sum_{i \in \mathcal{I}} \frac{P_{i}\left(x^{\prime}\right)}{P_{0}\left(x^{\prime}\right)}\right] \pi\left(x^{\prime} \mid x, 0\right) .
$$

For simplicity, denote the $X \times 1$ vector $\left\{Z_{0}(x)\right\}_{x \in \mathcal{X}}$ as $\mathbf{Z}_{0}$; write the $X \times X$ matrix $\pi\left(x^{\prime} \mid x, 0\right)$ as $\mathbf{\Pi}_{0}$, and write the $X \times 1$ vector $\left\{\ln \left[\sum_{i \in \mathcal{I}} \frac{P_{i}\left(x^{\prime}\right)}{P_{0}\left(x^{\prime}\right)}\right]\right\}_{x \in \mathcal{X}}$ as $\mathbf{m}$. The above equation can be written as

$$
\mathbf{Z}_{0}=\delta \mathbf{\Pi}_{0}\left(\mathbf{Z}_{0}+\mathbf{m}\right) .
$$

Thus,

$$
\mathbf{Z}_{0}=\left(\mathbf{I}-\delta \mathbf{\Pi}_{0}\right)^{-1} \delta \mathbf{m}
$$

Given this unique solution of $\mathbf{Z}_{0}$, (23) immediately provides $Z_{i}(x)$ for all $i \in \mathcal{I} /\{0\}$ and all $x \in \mathcal{X}$. To obtain $u_{i}(x)$ for $i \in \mathcal{I} \backslash\{0\}$, note that (22) implies that

$$
\mathbf{u}_{i}=\mathbf{Z}_{i}-\delta \mathbf{\Pi}_{i} \mathbf{Z}_{0}-\delta \boldsymbol{\Pi}_{i} \mathbf{m}
$$

where $\mathbf{Z}_{i}$ and $\mathbf{u}_{i}$ are $X \times 1$ vectors of $\left\{Z_{i}(x)\right\}_{x \in \mathcal{X}}$ and $\left\{u_{i}(x)\right\}_{x \in \mathcal{X}}$ respectively, $\Pi_{i}$ is the $X \times X$ matrix $\pi\left(x^{\prime} \mid x, i\right)$. Recall that in the standard exponential discounting model we have $Z_{i}(x)=$ $V_{i}(x)$, thus we can conclude that,$\left\{\mathbf{u}_{i}\right\}_{i \in \mathcal{I} \backslash\{0\}}$ and $\left\{\mathbf{V}_{i}\right\}_{i \in \mathcal{I}}$ are identified once $\delta, G$ and $\left\{u_{0}(x)\right\}_{x \in \mathcal{X}}$ are fixed. This replicates the proof of Proposition 2 in Magnac and Thesmar (2002). ${ }^{14}$

\section{Estimation Strategies}

In this section, we describe two estimation strategies based on our discussion about identification above. Both estimation strategy involves two steps, and they share the same first step. In the first step, we estimate from the data the choice probabilities $P_{i}(x)$ for all $i \in \mathcal{I}$ and all $x \in \mathcal{X}$, as well as the state transition probabilities $\pi\left(x^{\prime} \mid x, i\right)$ for all $i \in \mathcal{I}$ and all $\left(x^{\prime}, x\right) \in \mathcal{X}^{2}{ }^{15}$

\footnotetext{
${ }^{14}$ The only difference is that our argument above indicates that $\mathbf{V}_{0}$ does not have to be fixed. It can be identified from the model.

${ }^{15}$ It is useful to note that so far, our discussion has focused on short-panel (two period) data sets under stationarity assumption. Having two-period data allows one to non-parametrically estimate the transition probabilities $\pi\left(x^{\prime} \mid x, i\right)$; stationarity ensures that looking at a two-period slice of a potentially long panel is sufficient. Fang and Silverman (2006) considered a case without stationarity (specifically a finite horizon model) and showed that $\beta$ and $\delta$ could be poten-
} 
The second step is different for the two estimation strategies. The first estimation strategy involves two loops. In the inner loop, we solve equation system (19) and (22) for $u_{i}(x)=u_{i}\left(x_{r}, x_{e}\right)$ for a given triple of values for $\langle\beta, \tilde{\beta}, \delta\rangle$ for all $i \in \mathcal{I} \backslash\{0\}$ and all $x \in \mathcal{X}$ where we normalize $u_{0}(x)=0$ for all $x \in \mathcal{X}$. In the outer loop, we exploit the identifying assumption 5 in a straightforward manner. We know that at the true values of $\langle\beta, \tilde{\beta}, \delta\rangle$, for all $\tilde{x}_{e} \in \mathcal{X}_{e}$, the standard deviation of $\hat{u}_{i}\left(x_{r}, \tilde{x}_{e}\right)$ with respect to $\tilde{x}_{e}$ should be 0 , because Assumption 5 requires that $u_{i}\left(x_{r}, \tilde{x}_{e}\right)$ does not depend on $\tilde{x}_{e}$. Therefore the estimator for $\langle\beta, \tilde{\beta}, \delta\rangle$ in our first estimation strategy is:

$$
\langle\beta, \tilde{\beta}, \delta\rangle=\arg \min _{\{\beta, \tilde{\beta}, \delta\}} \frac{1}{\left|\mathcal{X}_{r}\right|} \sum_{x_{r} \in \mathcal{X}_{r}}\left|\operatorname{std}\left[\hat{u}_{i}\left(x_{r}, \tilde{x}_{e} ;\langle\beta, \tilde{\beta}, \delta\rangle\right)\right]\right|
$$

where $\operatorname{std}\left[\hat{u}_{i}\left(x_{r}, \tilde{x}_{e} ;\langle\beta, \tilde{\beta}, \delta\rangle\right)\right]$ is simply the sample standard deviation of $\hat{u}_{i}\left(x_{r}, \tilde{x}_{e}\right)$, calculated under given values of $\langle\beta, \tilde{\beta}, \delta\rangle$, with respect to $\tilde{x}_{e}$.

The second estimation strategy is a maximum pseudo-likelihood estimation. As in the first estimation strategy, we first solve equation system (19) and (22) for $u_{i}(x)=u_{i}\left(x_{r}, x_{e}\right)$ for a given triple of values for $\langle\beta, \tilde{\beta}, \delta\rangle$ for all $i \in \mathcal{I} \backslash\{0\}$ and all $x \in \mathcal{X}$ where we normalize $u_{0}(x)=0$ for all $x \in \mathcal{X}$. Then we directly impose, by Assumption 5, the restriction that

$$
\hat{u}_{i}\left(x_{r}\right)=\frac{1}{\left|\left\{\left(x_{r}, \tilde{x}_{e}\right): \tilde{x}_{e} \in \mathcal{X}_{e}\right\}\right|} \sum_{\left\{\left(x_{r}, \tilde{x}_{e}\right): \tilde{x}_{e} \in \mathcal{X}_{e}\right\}} \hat{u}_{i}\left(x_{r}, \tilde{x}_{e}\right)
$$

where $\mathcal{X}_{e}$ is the set of possible values for the payoff irrelevant state variables $x_{e}$ we discussed in Assumption 5. Given $\hat{u}_{i}\left(x_{r}\right)$ as defined by (26), we can then use the model to predict the implied choice probabilities $\hat{P}_{i}(x ;\langle\beta, \tilde{\beta}, \delta\rangle)$, and formulate the pseudo-likelihood of the observed data, which can be written as:

$$
\mathcal{L}=\Pi_{n \in \mathcal{N}} \Pi_{i=1}^{I} \Pi_{x \in \mathcal{X}} \hat{P}_{i}(x ;\langle\beta, \tilde{\beta}, \delta\rangle)^{D_{i}(x)}\left[1-\hat{P}_{i}(x ;\langle\beta, \tilde{\beta}, \delta\rangle)\right]^{1-D_{i}(x)}
$$

where in the likelihood function, $n$ stands for an individual and $\mathcal{N}$ stands for the set of individuals in the sample, and also note that we partialed out the contribution from the state transitions $\pi\left(x^{\prime} \mid x, i\right)$ in the likelihood since it is already estimated in step one. We then maximize the pseudolikelihood function to estimate $\langle\beta, \tilde{\beta}, \delta\rangle$.

tially distinguished without exclusion restriction if the researcher has access to at least three-period panel data. Also, see Section 6 for discussions related to non-stationariy, longer panels and unobserved state variables or unobserved heterogeity. 


\subsection{Discussions}

Now we discuss a few practical issues that may arise in the estimation.

Solutions to the Equation System (19) and (22). Even though the equation system (19) and (22) have the same number of equations and unknowns, there is no guarantee that the system will have a unique solution for any arbitrary values of $\langle\beta, \tilde{\beta}, \delta\rangle \cdot{ }^{16}$ In practice, we deal with the potential no solution, or multiple solution problem as follows. If for a particular combination of $\langle\beta, \tilde{\beta}, \delta\rangle$, we are unable to find a solution to the equation system (19) and (22), we will simply set the objective $\sum_{x_{r} \in \mathcal{X}_{r}}\left|\operatorname{std}\left[\hat{u}_{i}\left(x_{r}, \tilde{x}_{e} ;\langle\beta, \tilde{\beta}, \delta\rangle\right)\right]\right|$ in (25) for estimation method 1 to $+\infty$ (i.e., a sufficiently large value), and the objective likelihood in (27) for estimation method 2 to $-\infty$ (i.e., a sufficiently small value). On the other hand, if for some particular combination of $\langle\beta, \tilde{\beta}, \delta\rangle$, we find multiple solutions to the equation system, we will, among all the solutions, pick the smallest values of the objective (25) for estimation method 1 and pick the highest likelihood in (27) for estimation method 2.

Size of the Support of exclusion Restriction State Variables $\mathcal{X}_{e}$. As we described in Section 3.2, the identification of the model comes from the presence of state variables that satisfy the exclusion restriction 5 . The exclusion variables identify $\langle\beta, \tilde{\beta}, \delta\rangle$ by requiring that only those combinations of the discount factors that can explain the observed data with payoffs $\left\langle\left\{u_{i}(x): i \in \mathcal{I} \backslash\{0\}, x \in \mathcal{X}\right\}\right\rangle$ that do not vary with respect to changes in $x_{e}$. Since we have three unknown discount factors $\langle\beta, \tilde{\beta}, \delta\rangle$, we need at least three cross- $x_{e}$ restrictions, i.e., a minimum of four different points in the support $\mathcal{X}_{e}$ are required. Also, the requirement for the size of the support $\mathcal{X}_{e}$ seems to differ between the two proposed estimation methods. In estimation method 1 , the larger the size of $\mathcal{X}_{e}$, the smoother the objective function $\sum_{x_{r} \in \mathcal{X}_{r}}\left|\operatorname{std}\left[\hat{u}_{i}\left(x_{r}, \tilde{x}_{e} ;\langle\beta, \tilde{\beta}, \delta\rangle\right)\right]\right|$ is with respect to $\langle\beta, \tilde{\beta}, \delta\rangle$. The effect of the size of $\mathcal{X}_{e}$ on the properties of the objective function in (27) is less pronounced.

Parametric Utility Function Specifications. Even though we described how $\left\langle\left\{u_{i}(x): i \in \mathcal{I} \backslash\{0\}, x \in \mathcal{X}\right\}\right\rangle$ can be non-parametrically solved from equation system (19) and (22) for given values of $\langle\beta, \tilde{\beta}, \delta\rangle$. In practice, we may often parameterize it as a function of observed state variables $x$, including the exclusion variables $x_{e}$. In such cases, the estimation methods described above can be adapted to minimize the standard deviation of the coefficient estimates for the exclusion variables $x_{e}$ for estimation method 1. Estimation method 2, though, still goes through unmodified (see Footnote 18 for an illustration in our Monte Carlo experiments).

\footnotetext{
${ }^{16}$ As we show in Section 3.3, the system does have a unique solution under the exponential discounting case, i.e., when $\tilde{\beta}=\beta=1$, for any $\delta \in(0,1)$.
} 


\subsection{Monte Carlo Experiments}

In this section we provide Monte Carlo evidence for the identification of discount factors in a dynamic discrete choice model using the two estimation methods described above. In this simple Monte Carlo exercise, we consider a binary choice decision problem, $i \in\{0,1\}$, facing an agent with infinite horizon, stationary state transition, and linear utility functions. There are two state variables $x_{r}$ and $x_{e}$. The state variable $x_{r} \in \mathcal{X}_{r}=\{0,1,2,3,4,5\}$, affects both instantaneous utility and state transition; while state variable $x_{e} \in \mathcal{X}_{e}=\{0,1,2,3\}$, affects only the state transition. ${ }^{17}$ We parameterize the instantaneous payoff functions $u_{i}\left(x_{r}\right)$ as follows: $u_{1}\left(x_{r}\right)=\alpha_{0}+\alpha_{1} x_{r}$; and normalize $u_{0}\left(x_{r}\right)=0$ for all $x_{r}$. The true parameters are set at: $\alpha_{0}=-0.1, \alpha_{1}=0.5, \delta=0.8$, $\beta=0.6$, and $\tilde{\beta}=0.7 .{ }^{18}$ For each sample size $(240,000,120,000$ and 12,500$)$, we randomly generate 2,000 simulation samples, and estimate the discount factors and corresponding utility parameters to examine the differences in performance of these two estimation methods.

Table 2 presents the Monte Carlo results. It shows that both estimation methods do a good job in recovering the true parameter values in large samples. Estimation results improve with increases in sample sizes. Because the second estimation strategy, the maximum pseudo-loglikelihood method, has only one loop in the second step, it is the method we use in our empirical exercise.

\section{An Empirical Application: Mammography Decisions}

In this section, we present an illustrative application of the identification and estimation method for $\langle\beta, \tilde{\beta}, \delta\rangle$ described in Sections 3.2 and 4 . We provide estimates of these key time preference parameters and then examine the role of present bias and naivety in women's decisions to undertake mammography.

\footnotetext{
${ }^{17}$ The state transition matrices are generated as follows. We first generate two random matrices $M_{0}$ and $M_{1}$ each with dimension $\# \mathcal{X}_{r} \times \# \mathcal{X}_{e}=6 \times 4=24$, with each entry a random number generated from a uniform [0, 1] distribution. We then normalize the entry in each row by its row sum to ensure a proper probability matrix. The resulting matrices are denoted $\Pi_{0}$ and $\Pi_{1}$, the choice-specific state transition probabilities. The $(j, k)$-th entry of matrix $\Pi_{i}$ where $(j, k) \in$ $\{1, \ldots, 24\}^{2}$ is the probability that $\left(x_{r}^{\prime}, x_{e}^{\prime}\right)$ takes on $k$-th combination condition on $\left(x_{r}, x_{e}\right)$ taking on $j$-th combination in this period and action chosen is $i \in\{0,1\}$. The matrices $\Pi_{1}$ and $\Pi_{0}$ are assumed to be known by the decision maker; and are directly taken to be the state transition probabilities in our Monte Carlo exercise reported in Table 2.

${ }^{18}$ Given the assumed linear utility functions in this Monte Carlo exercise, the objective function for the outer loop of step two of the first estimation strategy can be simplified to:

$$
\langle\beta, \tilde{\beta}, \delta\rangle=\arg \min _{\{\beta, \tilde{\beta}, \delta\}}\left\|\alpha_{2}\left(x_{e}\right)\right\| \text {. }
$$

where $\alpha_{2}$ is the estimated utility parameter for the exclusive restriction variables for a given combination $\langle\beta, \tilde{\beta}, \delta\rangle$ and ||.|| refers to the Euclidean metric.
} 


\begin{tabular}{|c|c|c|c|c|c|c|c|c|c|c|}
\hline \multicolumn{2}{|c|}{ Estimation Method } & \multicolumn{4}{|c|}{ Estimation Strategy I } & \multicolumn{5}{|c|}{ Estimation Strategy II } \\
\hline Parameters & $\delta$ & $\beta$ & $\tilde{\beta}$ & $\alpha_{0}$ & $\alpha_{1}$ & $\delta$ & $\beta$ & $\tilde{\beta}$ & $\alpha_{0}$ & $\alpha_{1}$ \\
\hline True Values & 0.8 & 0.6 & 0.7 & -0.1 & 0.5 & 0.8 & 0.6 & 0.7 & -0.1 & 0.5 \\
\hline \multicolumn{11}{|c|}{ Sample Size: 240,000} \\
\hline Mean & 0.837 & 0.633 & 0.691 & -0.098 & 0.499 & 0.815 & 0.588 & 0.696 & -0.100 & 0.500 \\
\hline Std. Dev. & 0.064 & 0.064 & 0.069 & 0.006 & 0.002 & 0.045 & 0.050 & 0.057 & 0.007 & 0.002 \\
\hline \multicolumn{11}{|c|}{ Sample Size: 120,000} \\
\hline Mean & 0.860 & 0.632 & 0.672 & -0.096 & 0.499 & 0.821 & 0.584 & 0.691 & -0.099 & 0.499 \\
\hline Std. Dev. & 0.066 & 0.079 & 0.079 & 0.008 & 0.003 & 0.053 & 0.078 & 0.077 & 0.009 & 0.003 \\
\hline \multicolumn{11}{|c|}{ Sample Size: 12, 500} \\
\hline Mean & 0.874 & 0.603 & 0.678 & -0.095 & 0.499 & 0.838 & 0.553 & 0.713 & -0.098 & 0.500 \\
\hline Std. Dev. & 0.104 & 0.162 & 0.119 & 0.020 & 0.008 & 0.107 & 0.160 & 0.123 & 0.020 & 0.009 \\
\hline
\end{tabular}

Table 2: Monte Carlo Results Under the Two Proposed Estimation Methods.

Notes: For each sample size, we generate 2000 random simulation samples. The Mean and Standard Deviations of the estimated parameters are with respect to the 2000 samples.

\subsection{Background on Mammography}

Among American women, breast cancer is the third most common cause of death, and the second leading cause of cancer death. According to American Cancer Society, from birth to age 39 , one woman in 231 will get breast cancer (<0.5\% risk); from age 40-59, the chance is 1 in 25 ( $4 \%$ risk); from age 60-79, the chance is 1 in 15 (nearly 7\%). Assuming that a woman lives to age 90, the chance of getting breast cancer over the course of an entire lifetime is 1 in 7 , with an overall lifetime risk of $14.3 \%{ }^{19}$

Breast cancer takes years to develop. Early in the disease, most breast cancers cause no symptoms. When breast cancer is detected at a localized stage before it spreads to the lymph nodes), the 5 -year survival rate is $98 \%$. If the cancer has spread to nearby lymph nodes (regional disease), the rate drops to $81 \%$. If the cancer has spread (metastasized) to distant organs such as the lungs, bone marrow, liver, or brain, the 5 -year survival rate is $26 \%$.

A screening, mammography, is the best tool available to find breast cancer before symptoms appear. Mammography can often detect a breast lump before it can be felt and therefore save lives by finding breast cancer as early as possible. For women over the age of 50, mammography

\footnotetext{
${ }^{19}$ As a useful comparison, breast cancer has a higher incidence rate than lung cancer in the US. In 2004, there were 217, 440 new cases for breast cancer in the U.S. (American Cancer Society).
} 
have been shown to lower the chance of dying from breast cancer by $35 \% .{ }^{20,}{ }^{21}$ Leading experts, the National Cancer Institute, the American Cancer Society, and the American College of Radiology recommend annual mammography for women over $40 .^{22}$ The guideline issued by the U.S. Preventive Services Task Force in 2002 also recommended mammography screening for women beginning at age 40 every $12-24$ months in order to reduce the risk of death from breast cancer. ${ }^{23}$

\subsection{Data}

The data used in this analysis are from the Health and Retirement Study (HRS). The HRS is a nationally representative biennial panel study of birth cohorts 1931 through 1941 and their spouses as of 1992. The initial sample includes 12,652 persons in about 7,600 households who have been interviewed every two years since 1992. The most recent available data are for year 2008 (wave 9). The survey history and design are described in more details in Juster and Suzman (1995). Since the HRS started asking women questions about their usage of mammography in 1996, our sample is limited to women interviewed in the HRS from 1996 to 2008 . We focus on the age group 51 to 64 , and exclude those observations with missing values for any of the critical variables. ${ }^{24}$ We also exclude those who have ever been diagnosed of (breast) cancer, ${ }^{25}$ since those who are diagnosed of cancer might be of a different group who do not make decisions on mammography or any other preventive health care the same way as do others. Our final sample consists of 12,307 observations (each observation is a two-period short panel pooled from two consecutive waves) for 7,021 individuals.

\footnotetext{
${ }^{20}$ Source: American Cancer Society.

${ }^{21}$ Also note that finding breast cancers early with mammography has also meant that many more women being treated for breast cancer are able to keep their breasts. When caught early, localized cancers can be removed without resorting to breast removal.

${ }^{22}$ See American Cancer Society website: http://www.cancer.org/Cancer/BreastCancer/DetailedGuide. Women age 40 and older should have a screening mammogram every year and should continue to do so for as long as they are in good health.

${ }^{23}$ The new guideline by the U.S. Preventive Service Task Force issued in November of 2009 changed its recommendation for women in their 40s. Now the guideline recommends that women in their 40s should not get routine mammograms for early detection of breast cancer. For women ages 50 to 74 , it recommends routine mammography screenings every two years. The American Cancer Society maintains its original recommendation that women from their $40 \mathrm{~s}$ should seek annual mammography.

${ }^{24}$ The key variables are education status, non-Hispanic white, education, self-reported health, insurance status, whether father and mother are alive or died after age 70, father and mothers education, income, and mammography usage (see Table 3 for their summary statistics).

${ }^{25}$ For those who entered in 1992 (i.e. the first wave), we know whether they have been diagnosed of breast cancer as of 1992. But for those who entered HRS in later waves, we only know whether any cancer has been diagnosed of since the survey from 1994 did not ask about breast cancer specifically.
} 


\subsection{Descriptive Statistics}

Table 3 provides summary statistics of the key variables for the sample we use in our empirical analysis. The sample of women we select are aged from 51 to 64 (note that we are combining twoperiod panels using individuals that appear in two consecutive samples), with an average age of 57.8. A large majority of the sample are non-Hispanic white $(80 \%)$ and with at least high school education (78\%). The average household income in our sample is about 49,680 dollars. $23 \%$ of the sample has a self-reported bad health and about $76 \%$ of the women undertook mammogram in the survey year. About 1.5\% of the women who were surveyed in a wave died within two years. $72 \%$ of our sample reported having at least one insurance plan. Finally, about $75 \%$ of the mothers (respectively, about $61 \%$ of the fathers) of the women in our sample are either still alive or died at age greater than 70 at the time of the interview. $43 \%$ of the mothers (respectively, about $40 \%$ of the fathers) of the women in our sample finished at least high school. From one wave to the next wave, there is slight increase in the fraction of survivors who report bad health (an increase from $23 \%$ to $25 \%$ ).

\subsection{Decision Time Line}

Figure 1 depicts the time line for mammography decisions for women in our sample. As we mentioned earlier, we only consider women who are alive and have not yet been diagnosed with any cancer (thus not breast cancer) in the first period. Given her period-1 state variables, she makes the decision of whether to undertake mammography. Mammography detects breast cancer with very high probability, though not for certain, if the woman has breast cancer. In the event that the woman has breast cancer, early detection of breast cancer will lead to higher survival probability.

To fully capture the diagnostic nature of mammography, we would need to have information about whether the woman has breast cancer at any period, and estimate the probability of detecting breast cancer with ( $p_{1}$ in Figure 1$)$ and without ( $p_{2}$ in Figure 1) mammogram. However, we do not have access to such data. In HRS, even though we have information on women's mammography choices from 1996 on and we know whether their doctors have told them that they have any cancer, we do not have information on which kind of cancer they have been diagnosed of. ${ }^{26}$

Due to these data issues, we decide to go directly from the mammography decision to the live/death outcome and health status if alive (see our empirical specification below), without going through the intermediate step (having breast cancer or not). That is, we simply capture the ultimate effect of undertaking mammography as to lower the probability of dying, and to lower

\footnotetext{
${ }^{26}$ Even for those over age 65 with matching Medicare claim data, the number of observations is not big enough for us to get the needed probabilities of being diagnosed with breast cancer with and without mammography stratified by all the state variables we want to control in our model such as age, race, health status, income, and education status.
} 


\begin{tabular}{llllll}
\hline \hline Variable & Mean & Std. Dev. & Min & Max & Obs. \\
\hline Mammogram & 0.760 & 0.427 & 0 & 1 & 12307 \\
Bad Health & 0.230 & 0.421 & 0 & 1 & 12307 \\
Married & 0.704 & 0.456 & 0 & 1 & 12307 \\
White (Non-Hispanic) & 0.797 & 0.402 & 0 & 1 & 12307 \\
High School & 0.775 & 0.417 & 0 & 1 & 12307 \\
Age & 57.82 & 3.95 & 51 & 64 & 12307 \\
Death & 0.015 & 0.120 & 0 & 1 & 12307 \\
Insurance & 0.720 & 0.449 & 0 & 1 & 12307 \\
Household Income (\$1000) & 49.68 & 68.34 & 0.101 & 2,136 & 12307 \\
Log of Household Income & 10.31 & 1.06 & 4.62 & 14.57 & 12307 \\
Mother Still Alive or Died After Age 70 & 0.750 & 0.432 & 0 & 1 & 12307 \\
Mother Education (High School or Higher) & 0.431 & 0.495 & 0 & 1 & 12307 \\
Father Still Alive or Died After Age 70 & 0.611 & 0.488 & 0 & 1 & 12307 \\
Father Education (High School or Higher) & 0.404 & 0.491 & 0 & 1 & 12307 \\
Bad Health $(t+1)$ & 0.249 & 0.432 & 0 & 1 & 12128 \\
Household Income $(t+1)$ (\$1000) & 48.75 & 178.70 & 0.103 & 17,600 & 12128 \\
Log of Household Income $(t+1)$ & 10.27 & 1.05 & 4.64 & 16.68 & 12128 \\
\hline \hline
\end{tabular}

Table 3: Summary Statistics of Key Variables in the Estimation Sample.

Note: The last three variables in the table are observed only for those who survive to the second period. 


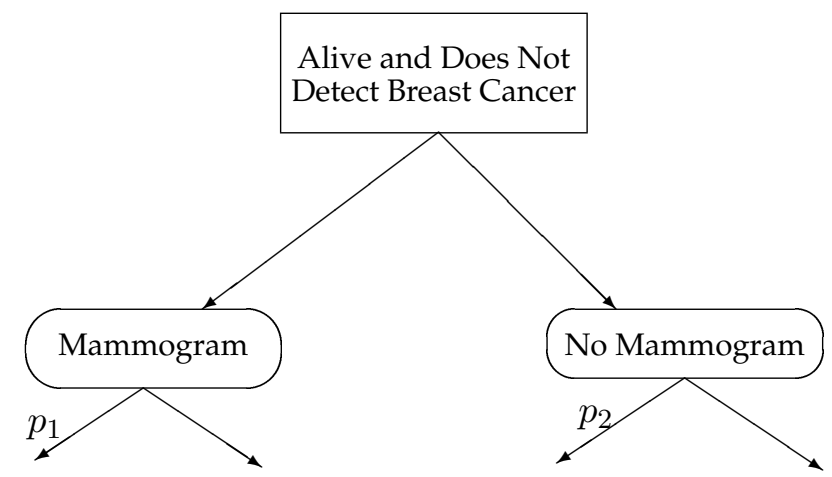

Detect Does Not Detect
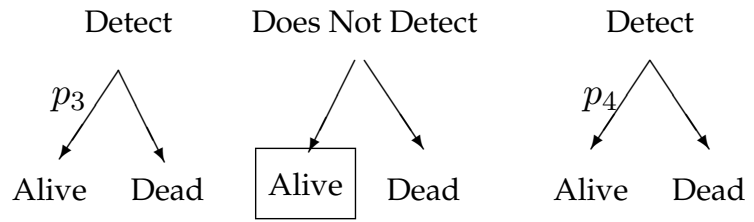

Does Not Detect

Figure 1: The Timeline for Mammography Decisions.

Notes: (1). $p_{1}>p_{2}$ : mammogram can detect breast cancer at its early stage; $p_{3}>p_{4}$ : survival rate is higher when breast cancer is detected at earlier stage.

(2). The states with rectangular framebox are those in which she will keep making decisions on whether to undertake mammography.

the probability of being in bad health status if alive.

\subsection{Empirical Specification}

For this application, we assume that each woman in our sample decides whether or not to undertake mammography each period ( $i=1$ if she does and $i=0$ if she does not). From the previous section on identification, we need to impose some normalization of the contemporaneous utilities. We normalize the individual's instantaneous utility at the death state to be zero. Note that no decision is necessary if one reaches the death state.

Now we describe the state variables we use in our empirical specification. They include: Age (AGE); Education Status (HIGHSCHOOL); Bad Health (BADHEALTH) which indicates whether the individual self reports bad health; Log of Per Capita Income (LOGINCOME), Death (DEATH) and whether her mother is still alive or died at age greater than 70 (MOTHER70).

If an individual stays alive, then we specify her utility from taking mammogram relative to not taking mammogram as:

$$
u_{1}(x)-u_{0}(x)=\alpha_{0}+\alpha_{1} \text { BADHEALTH }+\alpha_{2} \text { LOGINCOME }+\varepsilon_{t},
$$


where "BADHEALTH" is a binary variable indicating whether the agent is in bad health or not at time $t$; "LOGINCOME" denotes the logarithm of household income of the agent at time $t, \alpha_{0}$ and $\alpha_{1}$ are the utility parameters, and $\varepsilon_{t}$ denotes difference in the choice-specific utility shocks at time $t$. It is important to remark that even though the other state variables AGE, WHITE and HIGHSCHOOL do not show up in the above specification, it does not mean that these variables do not affect the instantaneous utility of the individual; what it means is that these variables affect the instantaneous utility under action 1 (mammogram) and action 0 (no mammogram) in exactly the same way.

The agents make their decisions about whether to get mammography by comparing the expected summations of current and discounted future utilities from each choice. Individuals are uncertain about their future survival probabilities, and if alive, the transition probabilities of future health and income. These probabilities depend on their choices about whether or not to get mammography; time-variant state variables including their lagged health status, their lagged income, and their age, denoted as AGE; and time-invariant state variables including their race, denoted by a binary variable WHITE, their education status HIGHSCHOOL, and the longevity of their mothers, denoted by the binary variable MOTHER70 which takes value 1 if the mother is still alive or died at the age older than 70 and 0 otherwise, and MOTHERHIGHSCHOOL (a binary variable which takes value 1 if mother finished high school or more, and 0 otherwise), or analogously, FATHER70 and FATHERHIGHSCHOOL (see Table 5 for the specifications).

Exclusion Variables. From the assumptions for exclusion restriction variables (see Assumption 5), we know that any variables that do not enter the relevant instantaneous payoff (after normalization), i.e. $u_{1}(x)-u_{0}(x)$, but affect the transition of payoff relevant state variables, can qualify as exclusion variables. Since only the variables BADHEALTH, LOGINCOME and DEATH enter the instantaneous payoff functions (after normalization), the other variables that affect the transition of the above three variables can all qualify as potential exclusion restriction variables, including Mother70, White, MARRIED, High_SCHOOL, AGE, or any other variables that one may find to have important effects on the transition of the state variables relevant to the instantaneous payoff functions, but do not directly affect the instantaneous payoff function (e.g., we experimented with insurance status in some of our specifications). ${ }^{27}$ In what follows, we report estimation results under two sets of exclusion variables. The sets of exclusion restriction variables are listed in the last panel in Table 6, and they differ in whether we use MOTHER70 and MOTHERHIGHSCHOOL or we use FATHER70 and FATHERHIGHSCHOOL. The first step estimates reported in Table 4 and

\footnotetext{
${ }^{27}$ See, e.g., Ayanian et al (1993) and Decker (2005) for the relationship between health insurance and health outcomes for women with breast cancer.
} 


\begin{tabular}{llllll}
\hline \hline \multirow{2}{*}{ Variable } & \multicolumn{2}{c}{$(1)$} & & \multicolumn{2}{c}{$(2)$} \\
\cline { 2 - 3 } \cline { 5 - 6 } \cline { 5 - 6 } BADHEALTH & Coeff. Est. & Std. Err. & & Coeff. Est. & Std. Err. \\
MARRIED & 0.049 & $(0.059)$ & & 0.022 & $(0.062)$ \\
WHITE & $0.220^{* * *}$ & $(0.058)$ & & $0.212^{* * *}$ & $(0.060)$ \\
INSURANCE & $-0.212^{* * *}$ & $(0.060)$ & & $-0.159^{* * *}$ & $(0.062)$ \\
HIGHSCHOOL & $0.351^{* * *}$ & $(0.048)$ & & $0.354^{* * *}$ & $(0.050)$ \\
LOGINCOME & $0.373^{* * *}$ & $(0.061)$ & & $0.347^{* * *}$ & $(0.063)$ \\
MOTHER70 & $0.318^{* * *}$ & $(0.027)$ & & $0.324^{* * *}$ & $(0.028)$ \\
MOTHERHIGHSCHOOL & $0.107^{* * *}$ & $(0.050)$ & & & \\
FATHER70 & $-0.106^{* *}$ & $(0.054)$ & & & \\
FATHERHIGHSCHOOL & \multicolumn{3}{c}{$0.081^{*}$} & $(0.048)$ \\
AGE & 0.008 & & & $0.093^{* * *}$ & $(0.051)$ \\
CONSTANT & $-3.032^{* * *}$ & $(0.451)$ & & $-3.185^{* * *}$ & $(0.471)$ \\
\hline Pseudo- $R^{2}$ & 0.0385 & & \multicolumn{2}{c}{0.0380} \\
\hline \hline
\end{tabular}

Table 4: Determinants of Mammography Decisions: The Choice Probabilities from Logit Regression.

Notes: ${ }^{*}{ }^{* *},{ }^{* * *}$ represents statistical significance at $10 \%, 5 \%$ and $1 \%$ respectively. Robust standard errors are presented.

5, as well as Figure 2, suggest that both mother and father side variables affect the transitions of the payoff relevant state variables.

\subsection{Estimation Results}

\subsubsection{First Step Estimates}

As we noted earlier, our estimation strategy has two steps. In the first step, we need to use the data to estimate choice probabilities, and the state transitions. Here we report these first-step estimation results. The choice probabilities and the death probability are estimated using Logit regressions; but the transition of BADHEALTH and LOGINCOME are estimated non-parametrically.

Logit Estimates of the Probability of Choosing Mammography as a Function of The State Variables. Table 4 produces the reduced form Logit regression results for the determinants of whether a woman will undertake mammogram in a given year under two specifications on the exclusion variables. Column (1) uses MOTHER70 and MOTHERHiGHSCHOOL together with other exclusion variables, while Column (2) uses FATHER70 and FATHERHIGHSCHOOL instead. In the 


\begin{tabular}{|c|c|c|c|c|}
\hline \multirow[t]{2}{*}{ Variable } & \multicolumn{2}{|c|}{ (1) } & \multicolumn{2}{|c|}{$(2)$} \\
\hline & Coeff. Est. & Std. Err. & Coeff. Est. & Std. Err. \\
\hline MAMMOGRAM & $-0.525^{* * *}$ & $(0.174)$ & $-0.498^{* * *}$ & $(0.182)$ \\
\hline BADHEALTH & $1.733^{* * *}$ & $(0.191)$ & $1.715^{* * *}$ & $(0.193)$ \\
\hline MARRIED & 0.105 & $(0.197)$ & 0.165 & $(0.202)$ \\
\hline WHITE & -0.132 & $(0.191)$ & -0.105 & $(0.202)$ \\
\hline INSURANCE & 0.209 & $(0.193)$ & $0.357^{*}$ & $(0.205)$ \\
\hline HIGHSCHOOL & 0.295 & $(0.198)$ & $0.400^{*}$ & $(0.206)$ \\
\hline LOGINCOME & $-0.240^{* * *}$ & $(0.082)$ & $-0.247^{* * *}$ & $(0.080)$ \\
\hline MOTHER70 & $-0.366^{* *}$ & $(0.180)$ & & \\
\hline MOTHERHIGHSCHOOL & 0.045 & $(0.194)$ & & \\
\hline FATHER70 & & & $-0.325^{*}$ & $(0.175)$ \\
\hline FATHERHIGHSCHOOL & & & -0.295 & $(0.206)$ \\
\hline AGE & $0.073^{* * *}$ & $(0.022)$ & $0.067^{* * *}$ & $(0.017)$ \\
\hline CONSTANT & $-6.609^{* * *}$ & $(1.557)$ & 5.20 & $(20.47)$ \\
\hline Pseudo- $R^{2}$ & \multicolumn{2}{|c|}{0.1073} & \multicolumn{2}{|c|}{0.1055} \\
\hline
\end{tabular}

Table 5: Determinants of Probability of Dying in Two Years.

Notes: ${ }^{*}{ }^{* *},{ }^{* * *}$ represents statistical significance at $10 \%, 5 \%$ and $1 \%$ respectively. Robust standard errors are presented.

specification reported in Column (1), we find that women who are married with higher household income, with high school education, and with health insurance are more likely to undertake mammograms, while white women are less likely than black women to undertake mammogram. All these coefficient estimates are significant at $1 \%$. Interestingly, we also find that women whose mothers are still alive or died after age 70 are less likely, but those whose mothers have at least high school are more likely to undertake mammograms. Finally, older women and women with bad health are more likely to undertake mammogram, though these coefficients are not statistically different from zero.

In the specification reported in Column (2), where we use the information about women's father as the exclusion variables, none of the other coefficient estimates change qualitatively. Interestingly we found that FATHER70 and FATHERHIGHSCHOOL both positively affect women's probability of undertaking mammogram. ${ }^{28}$

\footnotetext{
${ }^{28}$ Note that the coefficient estimates from such reduced form regressions, while informative, does not shed light on the mechanisms under which the observed relationships between a variable and the mammogram decision arises. For example, we see in Table 4 that women whose mother is still alive or died after age 70 are less likely to undertake mammogram. But it is not clear from the table why. The structural analysis we undertake below will help us to achieve
} 
Transition Probabilities of the Payoff Relevant State Variables: Determinants of the Probability of Dying in Two Years Table 5 reports the Logit regression results for the probability of dying in two years, again using two sets of exclusion variables, with Column (1) using MOTHER70 and MotherHighSCHOOL and Column (2) using FATHER70 and FATHERHighSCHOOL. The coefficient estimates show that undertaking mammogram significantly lowers the probability of death (notice that the average age of the sample is about 58 years). Not surprisingly, women with bad health are more likely to die, but mammogram reduces the probability of dying conditional on bad health. Also note that women whose mothers are either still alive or died after age 70 as well as women whose fathers are either still alive or died after age 70, are less likely to die, suggesting a genetic link of longevity between mothers as well as fathers and daughters.

Transition Probabilities of the Payoff Relevant State Variables: Evolution of Bad Health in Two Years Figure 2 depicts a subset of the results from the non-parametric estimation of the evolution of BADHEALTH for a selective combinations of the other state variables and the mammogram choice, for our exclusion restriction variable specification listed in Column (1) in Table 6. For example, Panel (a) shows that how the probability of having bad health in period 2 is affected by mammogram decision in the previous period and LOGINCOME, for women whose mothers are alive or died after age 70 and had bad health in the previous period. It shows that the probability of bad health decreases with Log Income, and is lower if one undertakes mammogram in the previous period. Similar negative relationship between the probability of bad health and income is also shown in other panels. Across all panels, we see that mammogram always reduces probability of bad health, and good health in the previous period predicts a higher probability of good health this period, and mother living or having died after 70 reduces the probability of bad health. A similar non-parametric estimation of LOGINCOME is also conducted.

\subsubsection{Second Step Estimates: Utility Parameters and Discount Factors}

Table 6 reports the estimation results for the parameters in the instantaneous utility function specification (28) and the identified discount factors $\delta, \beta$ and $\tilde{\beta}$, as well as standard errors calculated from the asymptotic distributions of the maximum pseudo-likelihood estimator. We report results from two specifications using different sets of exclusion restriction variables. In the specification reported in Column 1, we include only WHITE, AGE, MARRIED, HigHSCHOOL, INSURANCE, Mother70, and MotherHighSchoOL as the exclusion restriction variables, while in the specification reported in Column 2, we replace MOTHER70, and MOTHERHighSCHOOL by FATHER70, and FATHERHIGHSCHOOL.

a better understanding of the observed reduced form relationship reported in Table 4. 

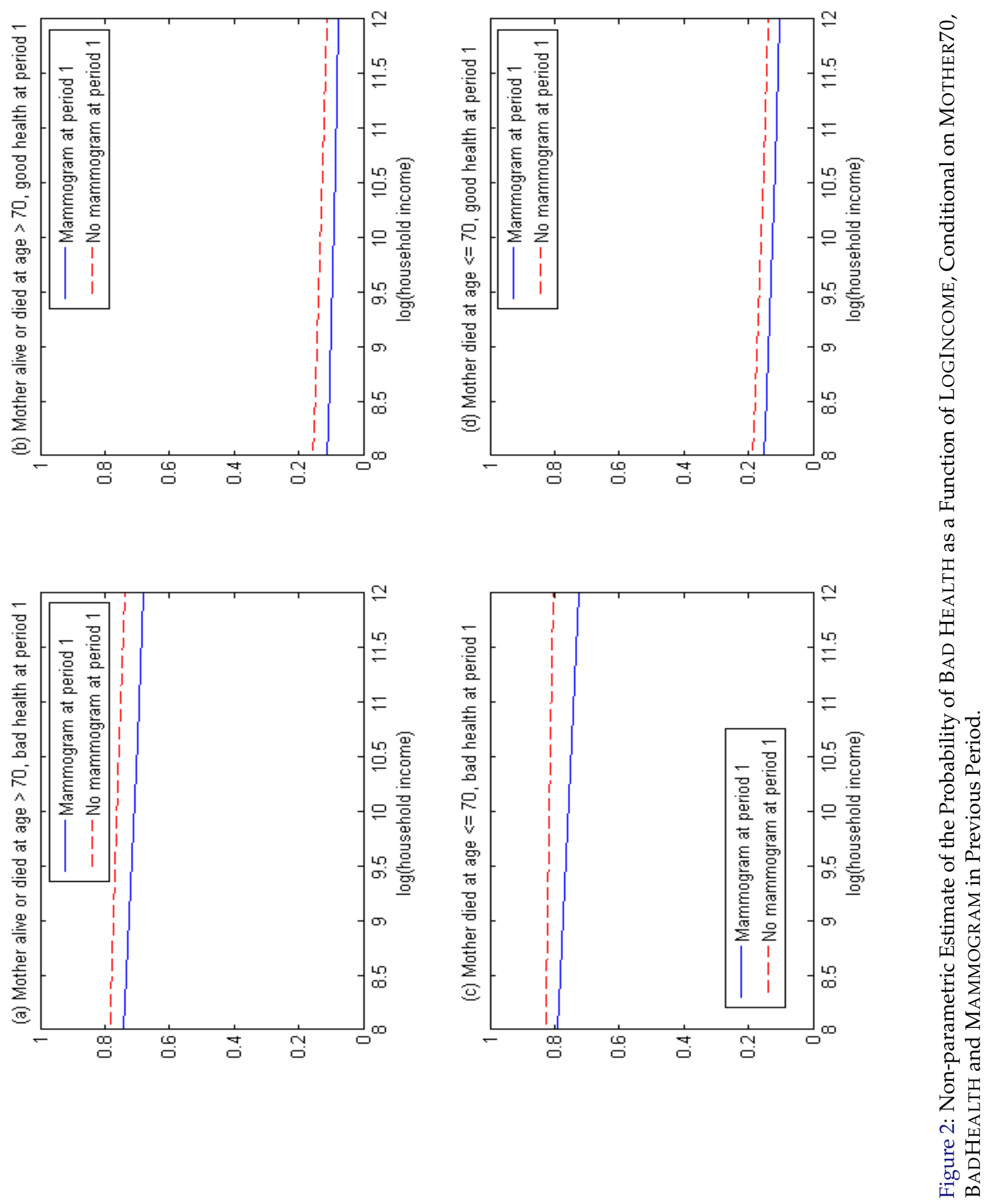
(1)

(2)

\begin{tabular}{|c|c|c|}
\hline \multicolumn{3}{|c|}{ Panel A: Instantaneous Utility Function Parameters } \\
\hline RАDHЕАтН & $-0.278^{* * *}$ & $-0.443^{* * *}$ \\
\hline ВАDНЕАLIH & $(0.084)$ & $(0.102)$ \\
\hline \multirow{2}{*}{ LOGINCOME } & $1.205^{* * *}$ & $1.278^{* * *}$ \\
\hline & $(0.060)$ & $(0.034)$ \\
\hline \multirow{2}{*}{ CONSTANT } & -1.035 & $-2.079^{* * *}$ \\
\hline & $(0.205)$ & $(0.026)$ \\
\hline
\end{tabular}

\begin{tabular}{lll}
\hline \multicolumn{4}{c}{ Panel B: Time Preference Parameters } \\
\hline \multirow{2}{*}{$0.722^{* * *}$} & $0.795^{* * *}$ \\
& $(0.089)$ & $(0.026)$ \\
$\beta$ & $0.718^{* * *}$ & $0.752^{* * *}$ \\
& $(0.070)$ & $(0.019)$ \\
$\tilde{\beta}$ & $0.9999^{* * *}$ & $0.9999^{* * *}$ \\
& $(0.060)$ & $(0.000)$ \\
\hline
\end{tabular}

Panel C: Hypothesis Tests

\begin{tabular}{lcc}
\hline $\mathrm{H}_{0}: \beta=1$ & Reject & Reject \\
$\mathrm{H}_{0}: \tilde{\beta}=\beta$ & Reject & Reject \\
\hline \multicolumn{2}{l}{ Exclusion Restriction Variables: } & \\
WHITE & Yes & Yes \\
AGE & Yes & Yes \\
MARRIED & Yes & Yes \\
HIGHSCHOOL & Yes & Yes \\
INSURANCE & Yes & Yes \\
MOTHER70 & Yes & No \\
MOTHERHIGHSCHOOL & Yes & No \\
FATHER70 & No & Yes \\
FATHERHIGHSCHOOL & No & Yes \\
\hline \hline
\end{tabular}

Table 6: Parameter Estimates for the Instantaneous Utility Function and Time Preference Parameters Under Two Sets of Exclusive Restriction Variables.

Notes: (1). The last panel indicates the exclusive restriction variables used in the specification in that column, with "Yes" meaning the variable is used, and "No" otherwise; (2). Standard errors for parameter estimates are in parenthesis, and ${ }^{* * *}$ indicates significance at $1 \%$ level; (3). For hypothesis tests reported in Panel C, all are rejected with $p$-value less than 0.01 . 
The parameter estimates, both for the instantaneous payoff function parameters and the time preference parameters, are quite similar both qualitatively and quantitatively, across the two specifications. We find that having bad health lowers the utility of undertaking mammography relative to not undertaking mammography. We also found that the relative utility of undertaking mammography increases with income, consistent with the finding in Table 4 that women with higher incomes are more likely to undertake mammography. The estimated negative constant term, though not statistically significant for specification 1 , is consistent with the idea that preventative health care typically involves huge one time instantaneous cost, which once combined with present bias and naivety about present bias might lead to undesirable health-related decisions.

More interestingly, we estimate $\delta, \beta$ and $\tilde{\beta}$ to be $0.722,0.718$ and 0.9999 respectively in specification 1 and $0.795,0.752$, and 0.9999 respectively in specification 2 . These point estimates show that women exhibit substantial present bias $(\beta<1)$ as well as naivety about their present bias $(\tilde{\beta}>\beta)$ when making mammography decisions. In Panel $\mathrm{C}$, we reported the results from three hypothesis tests: the null hypothesis $\beta=1$ (no present bias) and $\tilde{\beta}=\beta$ (no naivety) are rejected under both specifications with $p$-value less than 0.01 .

It is also interesting to compare our estimates for $\beta \delta$ with what is in the literature. Using the estimates of $\beta$ and $\delta$ reported in Table 6 , we have that our estimates for $\beta \delta$ is equal to $0.718 * 0.722 \approx$ 0.518 under specification 1 and is equal to $0.752 * 0.795 \approx 0.598$ under specification 2 . This can be compared with the estimate in Fang and Silverman (2009) where they estimate $\beta$ to be 0.338 and $\delta$ to be 0.88 , with $\beta \delta \approx 0.30$ for a group of single mothers with dependent children. It is important to note that our sample period is two years, while Fang and Silverman (2009)'s sample period is one year; also the sample of women in this paper are older and have very different social economic status (e.g. education and income) from the sample in Fang and Silverman (2009).

\subsection{Counterfactual Experiments}

Table 7 reports the mammography compliance rates predicted by the model and implied by two counterfactual experiments where in experiment [1], we assess the mammography rates predicted by the model if we hypothetically set $\tilde{\beta}$ equal to the estimated $\hat{\beta}$, and in experiment [2], we set both $\tilde{\beta}$ and $\beta$ to 1 . Experiment [1] allows us to assess the impact of naivety on mammography take-up rate, while experiment [2] allows us to assess the impact of both present bias and the naivety about present bias. If women in our sample are present-biased but fully sophisticated (i.e. in experiment 1 ), then the mammography compliance rate increases from $76.12 \%$ in the data to $77.96 \%$, and both are which is very similar to a baseline model predicted compliance rate $(76.09 \%)$. If, however, we have a case where the agents are exponential discounters (i.e., in experiment 2), that is, they do not have time-inconsistent preference, then the compliance rate goes up 


\begin{tabular}{lcc}
\hline \hline & \multicolumn{2}{c}{ Exclusion Restriction Variables } \\
\cline { 2 - 3 } & $(1)$ & $(2)$ \\
\hline Data & 0.7612 & 0.7631 \\
Model & 0.7609 & 0.7794 \\
Counterfactual Experiments: & & \\
\hline [1] No Naivety: $\tilde{\beta}=\beta[=\hat{\beta}]$ & 0.7796 & 0.7874 \\
[2] No Naivety and No Present Bias: $\tilde{\beta}=\beta=1$ & 0.8196 & 0.8099 \\
\hline \hline
\end{tabular}

Table 7: Mammography Compliance Rates Predicted by the Model and Implied by Different Counterfactual Experiments.

Notes: (1). The sample sizes slightly vary as we change the set of the exclusive restriction variables, which explains the changes in the mommography compliance rates in the data; (2). The exclusive restriction variables used in Columns 1 and 2 corresponds to those used in specifications 1 and 2 in Table 6 respectively.

from $76.12 \%$ to $81.96 \%$, which represents a $23.5 \%(=[(1-0.7612)-(1-0.8196)] /(1-0.7512))$ reduction in the mammography noncompliance rate. Thus, time-inconsistent preferences caused by present-bias and naivety about present-bias indeed have significant policy implications for the low compliance rates of preventive health care in the U.S.

\section{Conclusion and Discussions}

This paper extends the semi-parametric identification and estimation method for dynamic discrete choice models using Hotz and Miller's (1993) conditional choice probability (CCP) approach to the setting where individuals may have hyperbolic discounting time preferences and may be naive about their time inconsistency.

Our analysis showed that the three discount factors, the present bias factor $\beta$, the standard discount factor $\delta$ and the perceived present bias factor $\tilde{\beta}$ for naive agents can be separately identified. The key identifying restriction is that there exist variables that do not directly enter the instantaneous utility function but affect the transition of other payoff relevant state variables.

We proposed two estimation strategies based on the identification argument, and implement the proposed estimation method to the decisions of undertaking mammography to evaluate the importance of present bias and naivety in the under-utilization of mammography. Our estimates are consistent with the presence of both present bias and naivety about present bias. In our counterfactual experiments, we found that time-inconsistent preferences caused by present-bias and naivety about present-bias indeed have significant policy implications for the compliance rates of mammography take-up rates. 
In our paper, we also assume that the model is stationary. In many applications, such stationarity assumption may not be valid. However, we show in the Appendix that the identification arguments, properly modified, still work for the case of finite horizon models with non-stationary state transitions. Of course, estimating finite horizon models with non-stationary state transitions requires longer panels.

In this paper, we assumed that other than the choice-specific idiosyncratic payoff shocks, which we assume to be serially and cross-sectionally independent, we do not allow for any observed heterogeneity or unobserved state variables among individuals. Recent results by Kasahara and Shimotsu (2009) and Hu and Shum (2009) show that the conditional choice probabilities, which are crucial for implementing Hotz-Miller type estimators, can be identified in the presence of unobserved heterogeneity or unobserved state variables in the context of dynamic discrete choice models with exponential discounting. ${ }^{29}$ Whether their arguments still work for dynamic discrete choice models with hyperbolic discounting preferences remains an open question. Provided that conditional choice probabilities for any given state, including both observed and unobserved state variables, can be identified, the estimation methods we proposed here may be still valid. This is an important question we will examine in future research.

\section{References}

[1] Aguirregabiria, Victor, and Pedro Mira (2007a). “Sequential Estimation of Dynamic Discrete Games." Econometrica, Vol 70, 1519-1543.

[2] Aguirregabiria, Victor, and Pedro Mira (2007b). "Dynamic Discrete Choice Structural Models: A Survey." Working Paper 297, Department of Economics, University of Toronto.

[3] Ainslie, G., Picoeconomics: The Strategic Interaction of Successive Motivational States Within the Person (Cambridge [England]; New York: Cambridge University Press, 1992).

[4] Arcidiacono, P., H. Sieg, and F. Sloan (2007). “Living Rationally Under the Volcano? An Empirical Analysis of Heavy Drinking and Smoking." International Economic Review, 48(1), 37-65.

[5] Arcidiacono, P., and R. Miller (2007). "CCP Estimation of Dynamic Discrete Choice Models with Unobserved Heterogeneity." Working Paper.

\footnotetext{
${ }^{29}$ Arcidiacono and Miller (2007) proposed an EM algorithm to estimate such models with unobserved heterogeneity using conditional choice probabilities.
} 
[6] Ayanian, J. Z., B. A. Kohler, T. Abe, and A. M. Esptein (1993). “The Relation between Health Insurance Coverage and Clinical Outcomes among Women with Breast Cancer." The New England Journal of Medicine, 329(5), 326-331.

[7] Bajari, Patrick, C. Lanier Benkard and Jonathan Levin (2007). "Estimating Dynamic Models of Imperfect Competition." Econometrica, vol. 75, No. 5, 1331-1370.

[8] Bajari, Patrick, Han Hong and Denis Nekipelov (2010). "Game Theory and Econometrics: A Survey of Some Recent Research." Econometric Society World Congress in Shanghai.

[9] Barro, Robert J., "Ramsey Meets Laibson in the Neoclassical Growth Model," Quarterly Journal of Economics 114 (November 1999), 1125-1152.

[10] Carrillo, Juan D., and Thomas Mariotti, "Strategic Ignorance as a Self-Disciplining Device," Review of Economic Studies 67 (July 2000), 529-544.

[11] Chung, Doug, Thomas J. Steenburgh and K. Sudhir (2009). "Do Bonuses Enhance Sales Productivity? A Dynamic Structural Analysis of Bonus-Based Compensation Plans." Working Paper, Yale School of Management.

[12] Decker, S. L. (2005). "Medicare and the Health of Women with Breast Cancer." The Journal of Human Resources, 40(4), 948-968.

[13] Degnan, D., R. Harris, J. Ranney, D. Quade, J. A. Earp, and J. Gonzalez (1992). “Measuring the Use of Mammography: Two Methods Compared." American Journal of Public Health, 82(10), 1386-1388.

[14] Della Vigna, Stefano and Daniele Paserman (2005). "Job Search and Impatience." Journal of Labor Economics, 23(3), July, 527-588.

[15] Fang, Hanming, and Dan Silverman (2004). “On the Compassion of Time-Limited Welfare Programs." Journal of Public Economics, 88, 1445-1470.

[16] Fang, Hanming, and Dan Silverman (2006). "Distinguishing Between Cognitive Biases: Belief vs. Time Discounting in Welfare Program Participation." in Behavioral Public Finance, edited by Edward J. McCaffery and Joel Slemrod, Russell Sage Foundation.

[17] Fang, Hanming, and Dan Silverman (2009). "Time-inconsistency and Welfare Program Participation. Evidence from the NLSY." International Economic Review, Vol. 50, No. 4, 1043-1076.

[18] Gruber, J., and B. Koszegi (2001). "Is Addiction "Rational”? Theory and Evidence." Quarterly Journal of Economics, 116(4), 935-958. 
[19] Hausman, Jerry A. (1979). "Individual Discount Rates and the Purchase and Utilization of Energy-using Durables." Bell Journal of Economics, 10(1): 33-54.

[20] Hotz, Joseph, and Robert Miller (1993). “Conditional Choice Probabilities and Estimation of Dynamic Models."Review of Economic Studies, 60, 497-529.

[21] Juster, F. Thomas, and Richard Suzman (1995). "An Overview of the Health and Retirement Study." Journal of Human Resources, 30(5): S7-S56.

[22] Kasahara, H., and K. Shimotsu (2009). “Nonparametric Identification of Finite Mixture Models of Dynamic Discrete Choices." Econometrica, 77(1), 2009, 135-175.

[23] Krusell, Per, Burhanettin Kuruşçu and Anthony Smith, Jr., "Equilibrium Welfare and Government Policy with Quasi-Geometric Discounting," Journal of Economic Theory 105 (July 2002), $42-72$.

[24] Laibson, David (1997). “Gloden Eggs and Hyperblic Discounting." Quarterly Journal of Economics, 112(2), 443-477.

[25] Laibson, David, Andrea Repetto, and Jeremy Tobacman (2007). "Estimating Discount Functions with Consumption Choices Over the Lifecycle." Working Paper, Harvard University.

[26] Loewenstein, George, and Jon Elster, Choice Over Time (Russell Sage: New York, 1992).

[27] Mahajan, Aprajit and Alessandro Tarozzi (2010). “Time Inconsistency, Expectations and Technology Adoption: The Case of Insecticide Treated Nets." Work in Progress, Stanford and Duke University.

[28] Magnac, T., and D. Thesmar (2002). "Identifying Dynamic Discrete Decision Processes." Econometrica, 20(2), 801-816.

[29] Miller, Robert (1984). "Job Matching and Occupational Choice." Journal of Political Economy, 92(6), 1086-1120.

[30] O’Donoghue, T., and M. Rabin (1999a). “Doing It Now or Later." The American Economic Review, 89(1), 103-124.

[31] O’Donoghue, T., and M. Rabin (1999b), "Addiction and Self-Control." In Jon Elster, editor, Addiction: Entries and Exits, New York: Russell Sage.

[32] Pakes, Ariel (1986). "Patents as Options. Some Estimates of the Value of Holding European Patent Stocks." Econometrica, 54, 755-785. 
[33] Ariel Pakes and Paul McGuire (1994). “Computing Markov Perfect Nash Equilibrium: Numerical Implications of a Dynamic Differentiated Product Model." RAND Journal of Economics, Vol. 25, No. 4, 555-589.

[34] Pakes, Ariel, Michael Ostrovsky, and Steven Berry (2007). "Simple Estimators for the Parameters of Discrete Dynamic Games (with Entry/Exit Samples)." RAND Journal of Economics, Vol. 38, No.2, 373-399.

[35] Paserman, M. Daniele (2008). "Job Search and Hyperbolic Discounting: Structural Estimation and Policy Evaluation." Economic Journal, 118(531), 1418-1452.

[36] Pesendorfer, Martin, and Philipp Schmidt-Dengler (2003). "Identification and Estimation of Dynamic Games." NBER working paper 9726.

[37] Phelps, Edmund S., and Robert. A. Pollak (1968). “On Second-Best National Saving and Game-Equilibrium Growth."The Review of Economic Studies, 35(2), 185-199.

[38] Rust, John (1987). “Optimal Replacement of GMC Bus Engines. An Empirical Model of Harold Zurcher." Econometrica, 55(5), 999-1033.

[39] Rust, John (1994a). "Estimation of Dynamic Structural Models, Problems and Prospects: Discrete Decision Processes," in Christopher Sims and J.J. Laffont, eds., Proceedings of the 6th World Congress of the Econometric Society. Cambridge University Press.

[40] Rust, John (1994b). "Structural Estimation of Markov Decision Processes," in Robert Engle and Daniel McFadden eds., Handbook of Econometrics, Vol. IV. Amsterdam: North-Holland.

[41] Strotz, Robert H. (1956). "Myopia and Inconsistency in Dynamic Utility Maximization." The Review of Economic Studies, 23(3), 165-180.

[42] Warner, John T., and Saul Pleeter (2001). “The Personal Discount Rate: Evidence from Military Downsizing Programs." American Economic Review, 91(1): 33-53.

[43] Wolpin, Kenneth I. (1984). "An Estimable Dynamic Stochastic Model of Fertility and Child Mortality." Journal of Political Economy, 92, 852-874. 


\section{A Finite Horizon with Non-Stationary State Transition}

This Appendix discusses the identification strategy for a dynamic discrete choice model for (potentially naive) hyperbolic discounters with finite horizon and non-stationary state transitions. The time period in this case goes from $t$, the current period, until $T$, the end of horizon. In this section, we will use superscripts $t, t+1, t+2, \ldots, T$ to denote the time period for all the components in our analysis for clarification. The discussion in this section will be shorter and less detailed that that in Section 2, as will soon be clear that the identification strategy for this non-stationary case is essentially the same as the one for the stationary case, with only minor modification.

First, define the current choice-specific value function, $W_{i}^{t}\left(x^{t}\right)$, as follows:

$$
W_{i}^{t}\left(x_{t}\right)=u_{i}^{t}\left(x_{t}\right)+\beta \delta \sum_{x_{t+1} \in \mathcal{X}} V^{t+1}\left(x_{t+1}\right) \pi\left(x_{t+1} \mid x_{t}, i\right) d x^{t+1} .
$$

Then, define the choice-specific value function of the next-period self as perceived by the current self, $Z_{i}^{t+1}\left(x_{t+1}\right)$, as follows:

$$
Z_{i}^{t+1}\left(x_{t+1}\right)=u_{i}^{t+1}\left(x_{t+1}\right)+\tilde{\beta} \delta \sum_{x_{t+2} \in \mathcal{X}} V^{t+2}\left(x_{t+2}\right) \pi\left(x_{t+2} \mid x_{t+1}, i\right)
$$

Given $Z_{i}^{t+1}\left(x_{t+1}\right)$, we know that the current self's perception of her future self's choice, i.e., $\tilde{\sigma}$ as defined in Definition 2 is simply

$$
\begin{aligned}
\tilde{\sigma}\left(x_{t+1}, \varepsilon_{\mathbf{i}}^{\mathbf{t}+\mathbf{1}}\right) & =\max _{i \in \mathcal{I}}\left[u_{i}^{t+1}\left(x_{t+1}\right)+\varepsilon_{i, t+1}+\tilde{\beta} \delta \sum_{x_{t+2} \in \mathcal{X}} V^{t+2}\left(x_{t+2}\right) \pi\left(x_{t+2} \mid x_{t+1}, i\right)\right] \\
& =\max _{i \in \mathcal{I}}\left[Z_{i}^{t+1}\left(x_{t+1}\right)+\varepsilon_{i, t+1}\right] .
\end{aligned}
$$

Let us define the probability of choosing alternative $j$ by the the next period self as perceived by the current period self, $\tilde{P}_{j}\left(x_{t+1}\right)$, when next period state is $x_{t+1}$ :

$$
\begin{aligned}
\tilde{P}_{j}^{t+1}\left(x_{t+1}\right) & =\operatorname{Pr}\left[\tilde{\sigma}\left(x_{t+1}, \varepsilon_{t+1}\right)=j\right] \\
& =\operatorname{Pr}\left[Z_{j}^{t+1}\left(x_{t+1}\right)+\varepsilon_{j, t+1} \geq Z_{j^{\prime}}^{t+1}\left(x_{t+1}\right)+\varepsilon_{j^{\prime}, t+1}, \forall j^{\prime} \neq j\right] .
\end{aligned}
$$

Further denote

$$
V_{i}^{t+1}\left(x_{t+1}\right)=u_{i}^{t+1}\left(x_{t+1}\right)+\delta \sum_{x_{t+2} \in \mathcal{X}} V^{t+2}\left(x_{t+2}\right) \pi\left(x_{t+2} \mid x_{t+1}, i\right)
$$


According to the definition of $V(\cdot)$ as given by (2), $V^{t+1}\left(x_{t+1}\right)$ is simply the expected value of $\left[V_{i}^{t+1}\left(x_{t+1}\right)+\varepsilon_{i}^{t+1}\right]$ where $i$ is the chosen alternative according to $\tilde{\sigma}\left(x_{t+1}, \varepsilon_{t+1}\right)$. Thus it must satisfy the following relationship:

$$
V^{t+1}\left(x_{t+1}\right)=\mathrm{E}_{\boldsymbol{\varepsilon}^{\mathbf{t}+\mathbf{1}}}\left[V_{\tilde{\sigma}\left(x_{t+1}, \boldsymbol{\varepsilon}_{t+1}\right)}^{t+1}\left(x_{t+1}\right)+\varepsilon_{\tilde{\sigma}\left(x_{t+1}, \boldsymbol{\varepsilon}_{t+1}\right)}^{t+1}\right] .
$$

Now note from (A2) and (A3), we have

$$
V_{i}^{t+1}\left(x_{t+1}\right)=Z_{i}^{t+1}\left(x_{t+1}\right)+(1-\tilde{\beta}) \delta \sum_{x_{t+2} \in \mathcal{X}} V^{t+2}\left(x_{t+2}\right) \pi\left(x_{t+2} \mid x_{t+1}, i\right) .
$$

The relationship in (A5) is crucial as it allows us to rewrite (A4) as:

$$
\begin{aligned}
& V^{t+1}\left(x_{t+1}\right)=\mathrm{E}_{\varepsilon_{t+1}}\left[V_{\tilde{\sigma}\left(x_{t+1}, \varepsilon_{t+1}\right)}^{t}\left(x_{t+1}\right)+\varepsilon_{\tilde{\sigma}\left(x_{t+1}, \varepsilon_{t+1}\right), t}\right] \\
& =\mathrm{E}_{\boldsymbol{\varepsilon}_{t+1}}\left[\begin{array}{c}
Z_{i}^{t+1}\left(x_{t+1}\right)+\varepsilon_{\tilde{\sigma}\left(x_{t+1}, \boldsymbol{\varepsilon}^{\mathbf{t + 1}}\right), t} \\
+(1-\tilde{\beta}) \delta \sum_{x_{t+2} \in \mathcal{X}} V^{t+2}\left(x_{t+2}\right) \pi\left(x_{t+2} \mid x_{t+1}, \tilde{\sigma}\left(x_{t+1}, \varepsilon_{t+1}\right)\right)
\end{array}\right] \\
& =\mathrm{E}_{\varepsilon_{t+1}} \max _{i \in \mathcal{I}}\left[Z_{i}^{t+1}\left(x_{t+1}\right)+\varepsilon_{i, t+1}\right] \\
& +(1-\tilde{\beta}) \delta \mathrm{E}_{\varepsilon_{t+1}} \sum_{x_{t+2} \in \mathcal{X}} V^{t+2}\left(x_{t+2}\right) \pi\left(x_{t+2} \mid x_{t+1}, \tilde{\sigma}\left(x_{t+1}, \varepsilon_{t+1}\right)\right) \\
& =\mathrm{E}_{\varepsilon_{t+1}} \max _{i \in \mathcal{I}}\left[Z_{i}^{t+1}\left(x_{t+1}\right)+\varepsilon_{i, t+1}\right] \\
& +(1-\tilde{\beta}) \delta \sum_{j \in \mathcal{I}} \tilde{P}_{j}^{t+1}\left(x_{t+1}\right) \sum_{x_{t+2} \in \mathcal{X}} V^{t+2}\left(x_{t+2}\right) \pi\left(x_{t+2} \mid x_{t+1}, j\right)
\end{aligned}
$$

The probability of observing action $i$ being chosen at a given state variable $x$, in this nonstationary case, is still:

$$
P_{i}^{t}\left(x_{t}\right)=\operatorname{Pr}\left[W_{i}^{t}\left(x_{t}\right)+\varepsilon_{i, t}>\max _{j \in \mathcal{I} \backslash\{i\}}\left\{W_{j}^{t}(x)+\varepsilon_{j, t}\right\}\right]=\frac{\exp \left[W_{i}^{t}\left(x_{t}\right)\right]}{\sum_{j=0}^{I} \exp \left[W_{j}^{t}\left(x_{t}\right)\right]},
$$

where $P_{i}^{t}\left(x_{t}\right)$ is the current-period self's equilibrium choice probabilities and will be observed in the data.

The relationship between $W_{i}$ and $Z_{i}$ can no longer be described as in Equation 13, however, we still have

$$
\mathrm{E}_{\boldsymbol{\varepsilon}_{t+1}} \max _{i \in \mathcal{I}}\left\{Z_{i}^{t+1}\left(x_{t+1}\right)+\varepsilon_{i, t+1}\right\}=\ln \left\{\sum_{i \in \mathcal{I}} \exp \left[Z_{i}^{t+1}\left(x_{t+1}\right)\right]\right\}
$$


and

$$
\tilde{P}_{j}^{t+1}\left(x_{t+1}\right)=\frac{\exp \left[Z_{j}^{t+1}\left(x_{t+1}\right)\right]}{\sum_{i=0}^{I} \exp \left[Z_{i}^{t+1}\left(x_{t+1}\right)\right]} .
$$

Using (A8) and (A9), we can rewrite (A6) as

$$
\begin{aligned}
& V^{t+1}\left(x_{t+1}\right)=\ln \left\{\sum_{i \in \mathcal{I}} \exp \left[Z_{i}^{t+1}\left(x_{t+1}\right)\right]\right\} \\
& +(1-\tilde{\beta}) \delta \sum_{j \in \mathcal{I}} \frac{\exp \left[Z_{j}^{t+1}\left(x_{t+1}\right)\right]}{\sum_{i=0}^{I} \exp \left[Z_{i}^{t+1}\left(x^{t+2}\right)\right]} \sum_{x_{t+2} \in \mathcal{X}} V\left(x_{t+2}\right) \pi\left(x_{t+2} \mid x_{t+1}, j\right) .
\end{aligned}
$$

In this non-stationary case with finite horizon, at $t=T$ where the continuation value is zero, we have that

$$
Z_{i}^{T}=u_{i}^{T}=V_{i}^{T}
$$

which leads to:

$$
V^{T}=\ln \sum_{i \in \mathcal{I}} \exp \left[Z_{i}^{T}\right]=\ln \sum_{i \in \mathcal{I}} \exp \left[u_{i}^{T}\right]
$$

Equations (A11) and (A2) combined give us $Z_{i}^{T-1}$, which can be further combined with equations (A9) and (A10) for $t=T-1$ to give us $V^{T-1}$. We can keep doing backward induction in this way until we reach $V^{t+1}$, which can be used in (A1) to derive $W_{i}^{t}$, the current choice specific continuation value function. Equation (A7) shows the relationship between the observed choice pattern from the data and $W_{i}^{t}$. 